\title{
Blockchain-based Forward Supply Chain and Waste Management for COVID-19 Medical Equipment and Supplies
}

This paper was downloaded from TechRxiv (https://www.techrxiv.org).

LICENSE

CC BY 4.0

SUBMISSION DATE / POSTED DATE

$11-01-2021 / 16-01-2021$

\section{CITATION}

Ahmad, Raja Wasim; Salah, Khaled; Jayaraman, Raja; Yaqoob, Ibrar; Omar, Mohammed; Ellahham, Samer (2021): Blockchain-based Forward Supply Chain and Waste Management for COVID-19 Medical Equipment and Supplies. TechRxiv. Preprint. https://doi.org/10.36227/techrxiv.13553942.v1

DOI

10.36227/techrxiv.13553942.v1 


\title{
Blockchain-based Forward Supply Chain and Waste Management for COVID-19 Medical Equipment and Supplies
}

\author{
Raja Wasim Ahmad, Khaled Salah, Raja Jayaraman, Ibrar Yaqoob, Mohammed Omar, Samer Ellahham
}

\begin{abstract}
The year 2020 has witnessed unprecedented levels of demand for COVID-19 medical equipment and supplies. However, most of today's systems, methods, and technologies leveraged for handling the forward supply chain of COVID-19 medical equipment and the waste that results from them after usage are inefficient. They fall short in providing traceability, reliability, operational transparency, security, and trust features. Also, they are centralized that can cause a single point of failure problem. In this paper, we propose a decentralized blockchainbased solution to automate forward supply chain processes for the COVID-19 medical equipment and enable information exchange among all the stakeholders involved in their waste management in a manner that is fully secure, transparent, traceable, and trustworthy. We integrate the Ethereum blockchain with decentralized storage of interplanetary file systems (IPFS) to securely fetch, store, and share the data related to the forward supply chain of COVID-19 medical equipment and their waste management. We develop algorithms to define interaction rules regarding COVID-19 waste handling and penalties to be imposed on the stakeholders in case of violations. We present system design along with its full implementation details. We evaluate the performance of the proposed solution using cost analysis to show its affordability. We present the security analysis to verify the reliability of the smart contracts, and discuss our solution from the generalization and applicability point of view. Furthermore, we outline the limitations of our solution in form of open challenges that can act as future research directions. We make our smart contracts code publicly available on GitHub.
\end{abstract}

Index Terms-Blockchain, Ethereum, COVID-19, security analysis, forward supply chain, medical waste management.

\section{INTRODUCTION}

The world has experienced unprecedented crises in 2020 due to the COVID-19 pandemic. For example, worldwide trade disruptions, slower economic growth, shortage of medical supplies, equipment, and hospitals, and an overwhelmed medical waste generation in hospitals. During the COVID19 pandemic, a severe shortage of ventilators and personal safety equipment for medical staff has been reported in many hospitals in the USA [1]. A recent report reveals that the COVID-19 pandemic will cause the medical waste market

Raja Wasim Ahmad, Khaled Salah, and Ibrar Yaqoob are with the Department of Electrical Engineering and Computer Science, Khalifa University, Abu Dhabi 127788, United Arab Emirates.

Raja Jayaraman and Mohammed Omar are with the Department of Industrial and Systems Engineering, Khalifa University, Abu Dhabi 127788, United Arab Emirates.

Samer Ellahham is with Heart \& Vascular Institute, Cleveland Clinic Abu Dhabi, Abu Dhabi, United Arab Emirates.

Corresponding author: ibraryaqoob@ieee.org to grow beyond 16.62 billion by 2023 at a compound annual growth rate (CAGR) of $3.8 \%$ [2]. The world health organization (WHO) estimated that $15 \%$ of medical waste is hazardous that can be infectious, radioactive, or toxic to the environment. The handling of such infectious or toxic medical waste produced by hospitals requires caretakers to comply with safety rules and regulations during the handling of such materials. The key objectives of such rules and laws are to ensure the safety of frontline health workers. For instance, inappropriate handling of the unprotected needles that are used to treat infectious diseases such as HIV or COVID19 can cause the spreading of the disease [3]. Similarly, in the recent COVID-19 pandemic, improper disposal of the COVID-19 swab sticks or their use to test several patients can be dangerous since it can cause the spreading of the virus. Continuous monitoring of medical waste generated by COVID-19 infected patients and compliance of health provider's actions with set rules using blockchain technology can assist to efficiently handle medical waste. The data and transactions stored on the blockchain are accessible to the stakeholders involved in the forward supply chain and waste management processes through distributed public or private ledgers. The decentralization feature of blockchain increases the trust among stakeholders as it eliminates the need to assess the trustworthiness of the participants [4]-[6].

The forward supply chain ${ }^{1}$ involves the electronic ordering of medical equipment and supplies from manufacturing to consumption in the hospitals/COVID-19 testing centers. On the other hand, waste management of medical equipment and supplies refers to those processes that are involved in waste generation, collection, transportation, segregation, and disposal and recycling [7]. Traceability and operational transparency are the two main requirements of stakeholders involved in the modern forward supply chain and waste management practices of medical equipment [4]. The current COVID-19 medical equipment and waste are handled and managed by a vast network of manufacturers, distributors, retailers, shippers, storage facilities, suppliers, and waste treatment facilities who participate in the designing, production, shipment, sales, and disposal. The knowledge about what, where, and how the medical supplies and equipment are manufactured, originated, shipped, and used throughout their life cycle can significantly

\footnotetext{
${ }^{1}$ Note that our study does not focus on reverse supply chain processes as the COVID-19 related medical equipment and supplies (e.g., PPE, swab sticks and swab sample test tubes) are not recyclable to avoid the spreading of COVID-19.
} 


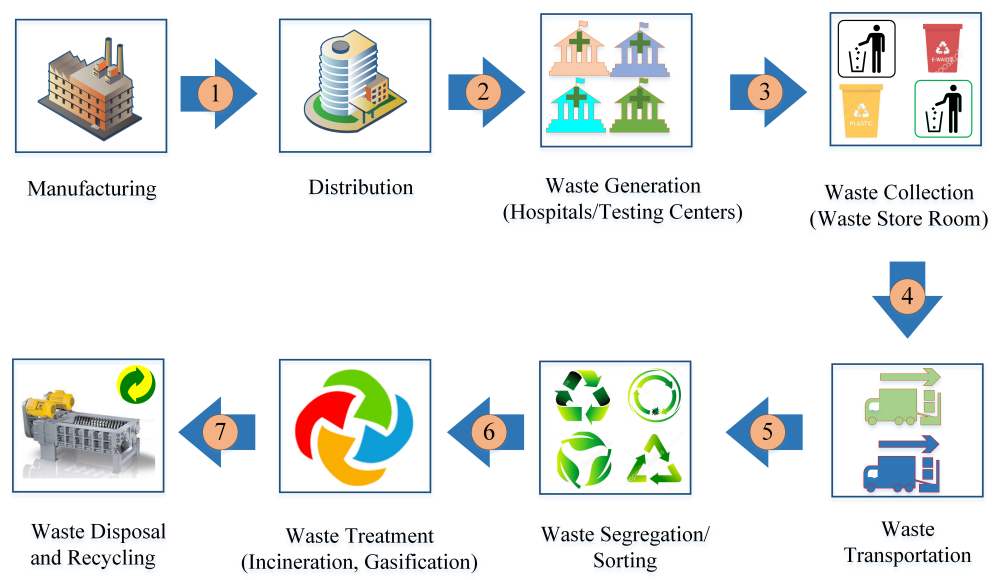

Figure 1: An overview of forward supply chain and waste management processes.

increase the trust among the involved stakeholders [4], [8]. On the other hand, the processes involved in waste management include collection, transportation, treatment, storage, disposal, and recycling of waste as highlighted in Fig.1. Modern waste disposal and treatment practices such as incineration, bioremediation, and plasma gasification efficiently and systematically handle the massive amount of generated waste to improve public health, environment, and climate. The increased rate of urbanization, economic development, the world population growth, and the rise in the standard of living in developing counties are major causes of the amount, rate, and variety of generated waste [9]. The transactions related to waste management and establishment of data provenance about the medical equipment and supplies can be stored on the blockchain, which might otherwise be reliant on centralized or paper-based systems that are not trustworthy and prone to a single point of failure or manipulation [4].

Most of the current technologies leveraged for managing forward supply chain and waste disposal are mainly based on cloud computing and the Internet of things (IoT) to cope with domestic, electronic, and biodegradable waste problems. For instance, the studies conducted in [10] and [11] employ IoT technology to monitor and report the available storage capacity of the waste bins deployed within a community. After collecting waste bin data using IoT sensors, it is stored on a central control server that processes it to identify and enlist those bins whose remaining storage capacity is below a certain threshold. It triggers a notification to the appropriate shipper through a route optimization algorithm to ship the waste. The existing cloud and the IoT-based systems used to manage the forward supply chain of medical equipment and their associated waste are incompetent in providing operational transparency, audit, verifiability, traceability, and identifying fraudulent documentation. Also, they are centralized that makes them vulnerable to a single point of failure problem. In this paper, we propose a blockchain-based solution that can manage the COVID-19 related medical equipment along with their supplies and waste disposal after usage. We implement safety rules to minimize the spreading of COVID-19. Through auditing service, our proposed solution issues penalties to the users for any non-compliance with COVID-19 related rules. The contributions of this paper are as follows:

- We showcase an Ethereum blockchain-based approach to trace and track the COVID-19 medical equipment and supplies and their waste after usage in a decentralized, secure, transparent, auditable, and trustful manner.

- We develop smart contracts along with algorithms to automate and implement the services for the forward supply chain management of COVID-19 related medical equipment and supplies, their waste shipment, and assurance of compliance with safety measures to minimize the spread of COVID-19. We employ decentralized storage of interplanetary file systems (IPFS) to overcome the blockchain storage limitations.

- We test and validate the proposed approach against many functionalities/services such as medical equipment order handling, ownership transformation, inventory management, waste shipment management, real-time waste monitoring, issuance of penalities to stakeholders to assure safety compliant operations, to assess their correctness, practicality, and feasibility. We report the logs on executing the transactions on the blockchain for each functionality.

- We present transaction execution and computational cost and security analysis of each function in the implemented smart contracts to demonstrate the affordability and reliability of the proposed solution. We outline several important challenges and limitations acting as future directions for new researchers.

- We propose a generic prototype solution that can be implemented on both public and private blockchains with minimal modifications based on the requirements and preferences of existing healthcare industries. Our implementation code can be publicly accessed via GitHub repository ${ }^{2}$.

The remainder of the paper is organized as follows. Section II discusses the related work. Section III presents the proposed system along with sequence diagrams to show the interactions

\footnotetext{
${ }^{2}$ https://github.com/AhmadKhalifaUniversity/Code/blob/main/Registration
} 
of stakeholders with the system. Section IV presents the implementation details. Section V tests and validates the system for a set of scenarios. Section VI presents a discussion on security and cost analysis and related research challenges. Section VII concludes the paper.

\section{RELATED WORK}

This section briefly discusses the recent developments and proposals related to the forward supply chain of medical equipment and waste management.

\section{A. Centralized Solutions for the Forward Supply Chain and Waste Management}

The forward supply chain processes of medical products (e.g., equipment, devices, and medicines) aim to deliver medical products timely and reliably to end users from the source. The technology-driven approaches including RFID tags, bar codes, near field communication (NFC), and serialization can assist to track the medical products throughout their forward supply chain phase in the healthcare industry [12]. In [13], an IoT-based assessment method is proposed that can track the medical devices in a hospital to establish an inventory system. It exploited the IoT technology to control the movement of the devices, Artificial Neural Network (ANN), and Fuzzy Logic (FL), to increase the visibility of the devices to optimize their supplies.

In [10], a prototype of an IoT-based system was developed that uses sensors' data to know the capacity of waste bins. This leads to define a transportation route of vehicles in collecting domestic waste from different geographical locations. The sensors attached to the waste bins continuously monitor and records the quantity of waste in waste bins to identify those waste bins that are full of waste. The data stored on a central server is processed to highlight whether or not a waste bin is eligible to be picked up by a waste collection service. The eligibility criteria introduced by the study states that the remaining storage capacity of the bin should be below $30 \%$ or the observed waste generation rate should be beyond a threshold limit for the past two hours. The authors used GIS data about 5000 waste bins located geographically at dispersed locations for estimating time and cost in collecting and disposing of waste. However, the study has overlooked the various critical aspects of waste management processes including waste segregation, recycling or incineration, and waste treatment.

A similar system developed in [11] has mounted several types of sensors inside the waste bins to monitor the level of solid waste and biogas inside the waste storing bins. It has employed the resource-rich cloud server to identify the waste bins with minimal remaining storage capacity by processing sensor data. The sensors are programmed to sense and send the data three times a day. Thus, it can assist to forecast the availability of the vehicle near the waste bin site. In [14], a cloud-storage based system to host waste data is presented. It employs sensors to monitor the waste bin's surroundings, and it immediately notifies the authorities about any illegal waste dumping attempt by the local community. The main building blocks of the proposed system include smart waste bins, driver modules, and a cloud based storage system. The smart waste bins are equipped with several sensors to store captured data on the cloud-based storage system. The driver module periodically notifies the shipper about the shortest available route based on the recent data for collecting the waste.

The researchers in [15] proposed a detailed waste management model highlighting the entities involved in the collection, transportation, processing, and recycling the organic, paper, plastic, metal, and toxic materials. It has employed several sensors to monitor the capacity, humidity, weight, chemical, and temperature of the bins storing solid waste. The study conducted in [16] has employed machine learning based analytics to dynamically schedule bin servicing by generating optimized routes to collect the bins. The above-mentioned studies have used centralized servers or cloud-based storage systems to store and process data collected by sensors. However, centralization can affect the privacy, security, transparency, and integrity of data. Blockchain, since it follows the decentralized storage and processing model, can bring about trust and transparency in existing waste management systems.

\section{B. Blockchain-based Solutions for the Forward Supply Chain and Domestic and Electronic Waste}

Blockchain-based solutions employed in the healthcare industry assist securely trace and track the medicines from manufacturer to distributors to patients. In [17], authors exploited the Gcoin blockchain to design a system that can trace the drugs throughout their supply chain process. It has digitized the operations involved in business processes among manufacturers, wholesalers, pharmacies, and drug consumers. The immutable logs of the transactions stored on blockchain enable authorities to identify the counterfeit medicines supplied to the hospitals. In [18], researchers have proposed a framework that can provide safety to drugs, and assures that the purchased medicines are manufactured by an approved manufacturer. Establishing provenance data about the medical equipment, similar to medical drugs, is the main concern of the authorities as safety and quality of medical equipment can affect human lives. In [19], [20], authors identified and presented the participants involved in blockchain-based systems to trace a medical device.

Traditional waste management systems do not offer incentives to people to produce less amount of waste than what they are producing. In [21], a flexible pricing and environmentally friendly waste management system named Smart waste management system (SWMS) was proposed. It introduces a weight-based price estimation model to charge the service cost to the waste producers. The SWMS employs IoT technology to monitor, measure, and collect waste-related data about waste bins located in different geographical regions. The proposed system offers a dual service model since it has hosted waste management services on both central servers and a blockchain network (Ethereum platform) to balance the throughput and reliability issues. The central servers are designated to provide services to those stakeholders (e.g., transportation companies) 
that need fast read/write operations. The blockchain was used to assist the users to make micropayments in a transparent and trusted way with the least financial transaction cost overhead. The drawback of the system is its incompetence to handle the man-in-middle and DoS attacks in centralized-based services.

In [22], an Ethereum-based system called Electronic waste management (EWM) was proposed that assures compliance with waste disposal guidelines for electrical and electronic equipment (EEE). The stakeholders considered by the proposed system for automating the EEE waste management services include producer, consumer, and retailer of electronic components, waste collection center, government authority, and recycling unit. The smart contracts calculate, record, report, and provide incentives to the consumer for returning the EEE waste to the retailer. The retailer-centered smart contracts verify that the waste is received for all sold EEE. The retailer ships the collected waste to the EEE producer and assures that a portion of the EEE original cost is paid to the consumer. The smart contracts calculate and impose the penalties to the producer if waste is not collected from retailers within the predefined period. However, this study overlooks the monitoring of waste during its transportation.

In [23], the authors proposed a 5G-enabled blockchainbased system that assists in tracking electronic products throughout their lifetime. The system employs an Ethereum blockchain platform to implement and present a working prototype of the system comprising of the manufacturer, supplier, retailer, customer, and E-waste facility unit. Smart contracts of the E-waste facility unit calculate and give incentives to customers for channelizing their waste to dispose of it at the E-waste facility. It allows registered government agencies (e.g, retailers) to collect and dispose of the waste of customers (Ewaste facility). It requires all stakeholders to deposit escrow (equivalent to the tax amount of the product) in a smart contract wallet to force them to comply with the set rules. The deposited amount along with incentives is returned to the stakeholders when waste reaches the facility after evaluating the stakeholder's compliance with rules. The performance assessment of the system in terms of system gas consumption and throughput was not discussed in the study.

In [24], a working prototype of an Ethereum-based system is proposed that assists the users in monitoring waste of solid materials (e.g., computers and smartphones). Unlike [22], [24] can continuously monitor and track waste on its transportation through trucks to the waste storage factory. Key external stakeholders of the architectural design of the proposed system include a collection manager, storage manager, transaction manager, and treatment manager. The collection manager generates and stores the $\mathrm{QR}$ code that refers to a package holding solid waste material on the blockchain. The transportation manager records the state, location, and route information about the vehicle transporting solid waste. Subsequently, the collected waste at the waste-storage unit is processed, and the treatment manager assigns tags to the treated waste. The proposed system has used off-chain storage systems to temporarily store encrypted data related to solid waste. The system can be made more proficient by employing multiple blockchain platforms to keep the balance between security and throughput.

In [25], a prototype of an Ethereum-based system that can track the waste across the border in a secure, tamper-proof, and privacy-preserving way was presented. The sensitive and non-disclosable information are stored locally to preserve the privacy of data. For audit-critical data such as e-waste type, assets, handler, and log of executed processes during e-waste processing, treatment, and handling, it digitally signs the data and transactions to further protect it. Through smart contracts, authorizes can audit and verify the reasoning of delay in reaching transportation between exchanged locations, the volume of transportation, and the validity of waste handling methods. This study can be further enhanced by introducing incentives and penalties into it to force the participating entities to observe the waste management rules. Another study presented in [26] has used a design science approach to highlight the main actors, use case scenarios, and processes involved in waste management across the borders.

The lack of operational transparency can cause frauds that occur during the waste collection stage. The study conducted in [27] proposes a framework for the traceability of personal protective equipment (PPE) for medical staff combating with COVID-19. In [28], the authors presented a system that uses an image analysis-based technique to identify the frauds related to waste collection. The proposed study calculates the similarity index between the two images that are captured at the garbage collection point and waste bin tray, estimate the weight of garbage, location, and time differences of two captured images, to identify waste-related frauds. After calculating such data, it incentivizes the users. It employed blockchain technology to securely transfer the cryptocurrency to the users as an incentive for collecting the waste. The proposed system can be extended by implementing object recognition techniques to identify the waste type (e.g., EEE). Another study conducted in [29] has proposed a blockchain-based system to keep track of all remanufacturing/refurbishing processes to smartphones. However, the authors have not discussed a solution to verify the frauds related to remanufacturing/refurbishing processes.

In summary, none of the aforementioned studies has highlighted the need for improved transparency, efficiency, and trust in the forward supply chain and waste management of COVID-19 medical equipment. In contrast, this research study proposes a blockchain-based solution that can assist the stakeholders to trace and track the medical equipment such as swab sticks and swab sample test tubes to verify their disposal in a secure, transparent, trustful, decentralized, and auditable way.

\section{PRoposed BlockChain-BASEd SOLUTION}

In this section, we present a high-level design of the proposed blockchain-based solution to manage COVID-19 medical equipment supplies and their waste after usage. Fig. 2 highlights the main stakeholders, system components, and actions of the stakeholders involved in the management of medical equipment and their waste part. Further discussion is provided in the following section. 


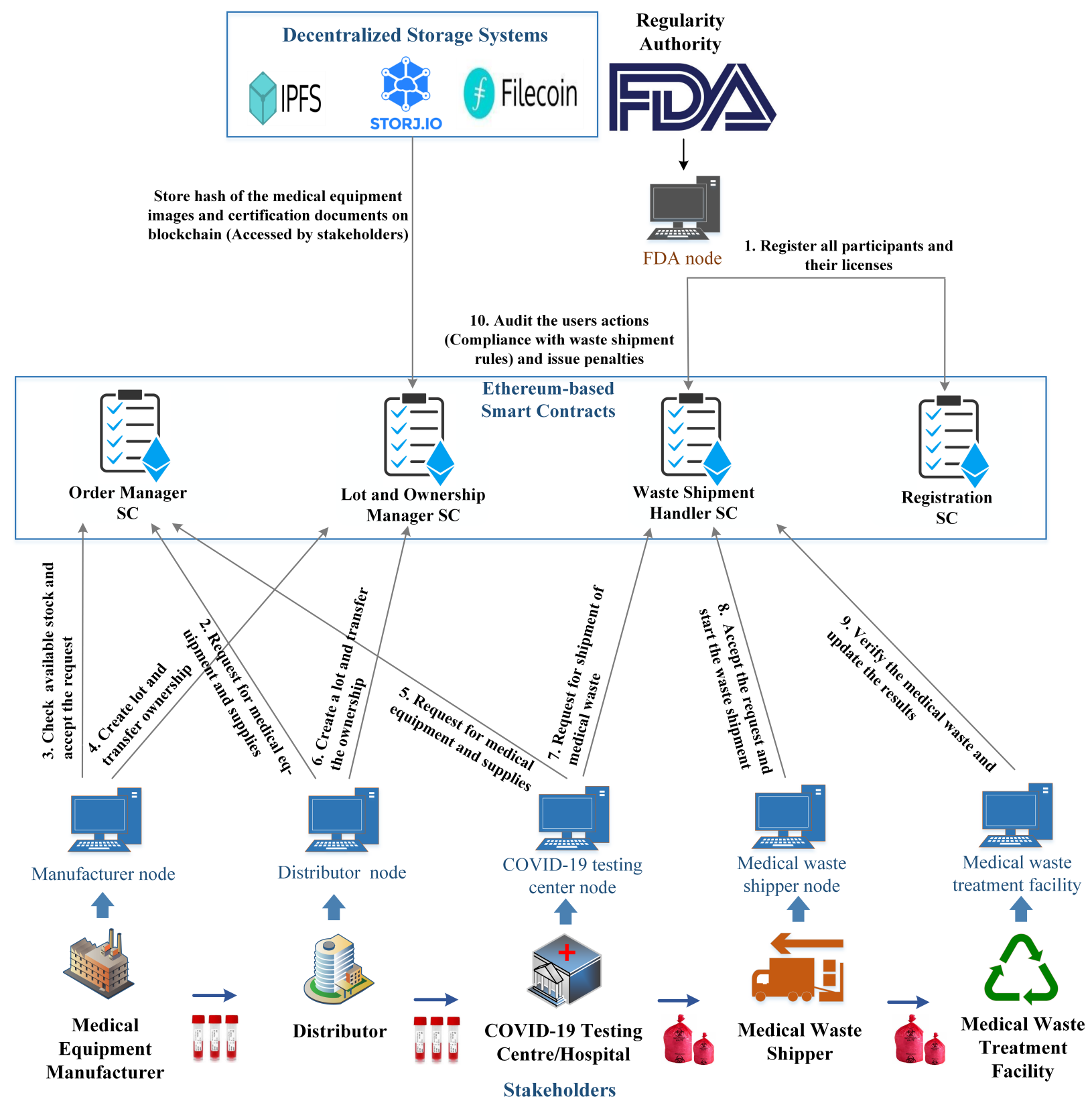

Figure 2: An overview of the proposed blockchain-based system for the forward supply chain and waste management of COVID-19 medical equipment.

\section{A. System Participants and Components}

Herein, we briefly explain the responsibilities of each participant involved in the forward supply chain of COVID-19 medical equipment and medical waste generation, handling, monitoring, and shipment. It also discusses the key components of the system that facilitates the secure sharing of data among participating stakeholders.

COVID-19 Medical Equipment Manufacturer: The role of a medical manufacturer is to store details about the newly manufactured medical equipment and supplies on the blockchain. The most important data about the manufactured medical equipment and supplies include product name, type, cost, credentials of the manufacturer, manufacturing date, design, composition, and certifying agency. A manufacturer normally researches the current need and requirements of the market to develop medical equipment. It surveys the market to find the trusted raw material supplier and places an order for the supply of raw material to manufacture medical equipment and equipment. It assures that all necessary documents such as specifications and certification records regarding all manufactured medical equipment are available. Also, the manufacturer should be genuine and all the details about the valid license should be publicly made available.

Distributor: The responsibility of the distributor in the forward supply chain of medical equipment and supplies used in COVID-19 screening centers is to revisit the market demand 


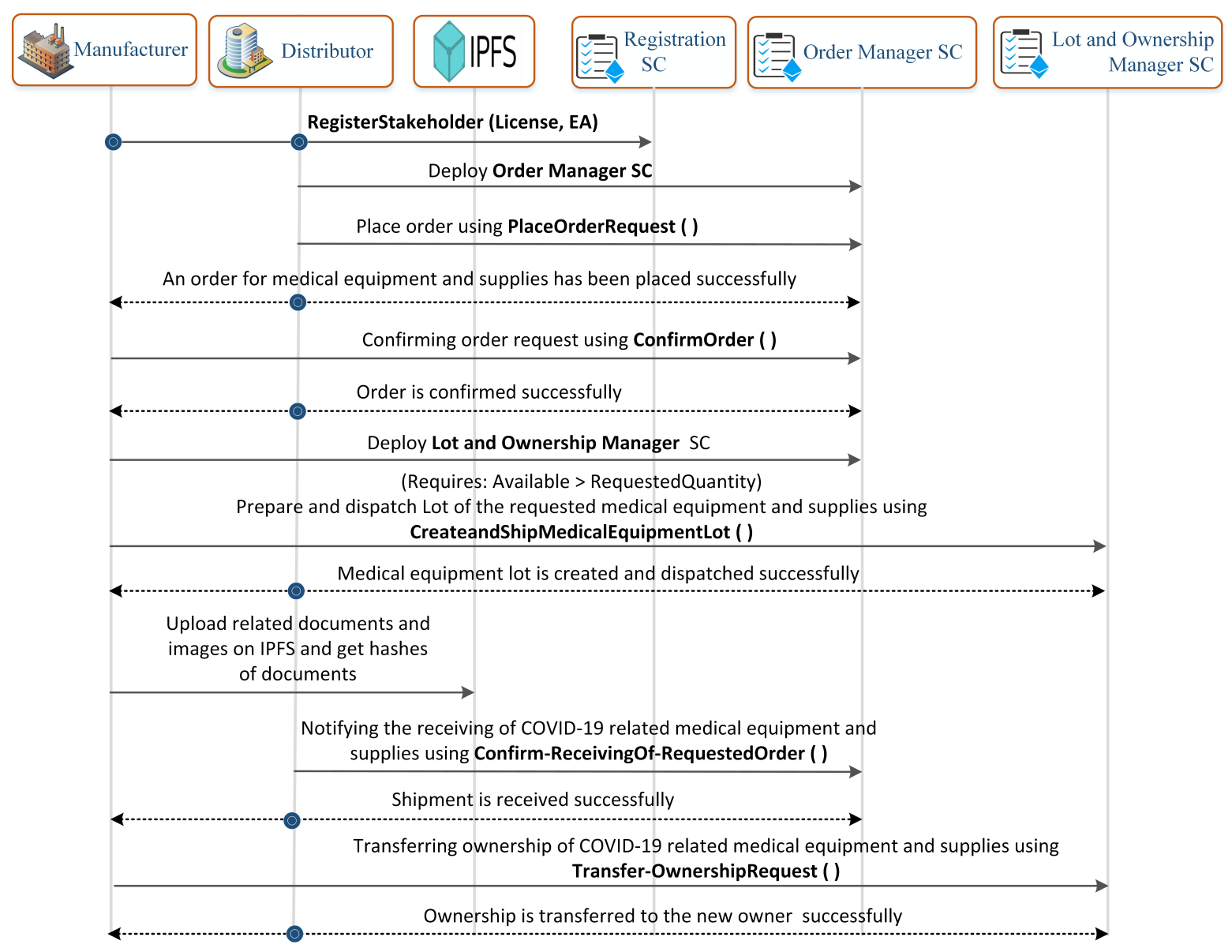

Figure 3: Sequence diagram showing the function calls and events between manufacturer and distributor.

to purchase equipment from the registered and highly reputed manufacturers. Thus, it acts as an intermediary between medical equipment consumers such as COVID-19 screening centers or hospitals and medical equipment manufacturers. A distributor aims that the order placed by the hospitals for the medical equipment and supplies should be immediately fulfilled. It is also responsible to create and prepare the lot of medical equipment and ship them to consignees. It needs to be ensured that the distributor should have a valid license to purchase and sell medical equipment and supplies.

COVID-19 Testing Center/Hospital: COVID-19 testing centers/Hospitals are one of the consumers of medical equipment and supplies (COVID-19 swab sticks). Medical equipment and supplies are normally purchased from the distributor or they are directly shipped from the manufacture. COVID-19 testing centers/Hospitals generate medical waste on testing the public for COVID-19 using medical equipment and supplies such as nasal swab sticks. The hospital staff temporarily stores medical waste in a safe storage room. The waste handlers and nursing staff makes sure that the waste is managed in a safe way to comply with standard operating procedures to deal with the COVID-19 pandemic. Later on, this medical waste is transported to the waste treatment facility for processing and disposal.

Medical Waste Shipper: The medical waste generated in
hospitals/COVID-19 testing centers is shipped to the waste treatment facility via third-party shipping services for treatment and disposal. To comply with the COVID-19 standard operating procedures, medical staff, and medical waste shippers having COVID-19 symptoms are not allowed to handle medical waste. However, those staff and shippers who have received COVID-19 vaccination can handle medical waste. Finally, it should be assured that the bags carrying COVID-19 medical waste are sealed properly, not mixed up with general waste, and secure enough against human interaction during its shipment.

Medical Waste Treatment Facility: The main responsibility of the manager in a medical waste treatment facility is to ensure safe interaction with medical waste during its treatment and disposal stage. At the medical waste treatment facility, COVID-19 medical waste is handled carefully especially during the waste segregation and processing phase to comply with the standard operating procedures devised to interact with COVID-19 waste. The medical equipment including nasal swab sticks and swab tubes are disposed of at the medical waste treatment facility since they are not recyclable, thereby minimizing the chances of spreading COVID-19 disease.

Regularity Authority: The responsibility of the regularity authority such as the Food and Drug Administration (FDA) is to register (on-chain) each stakeholder (license) along with 


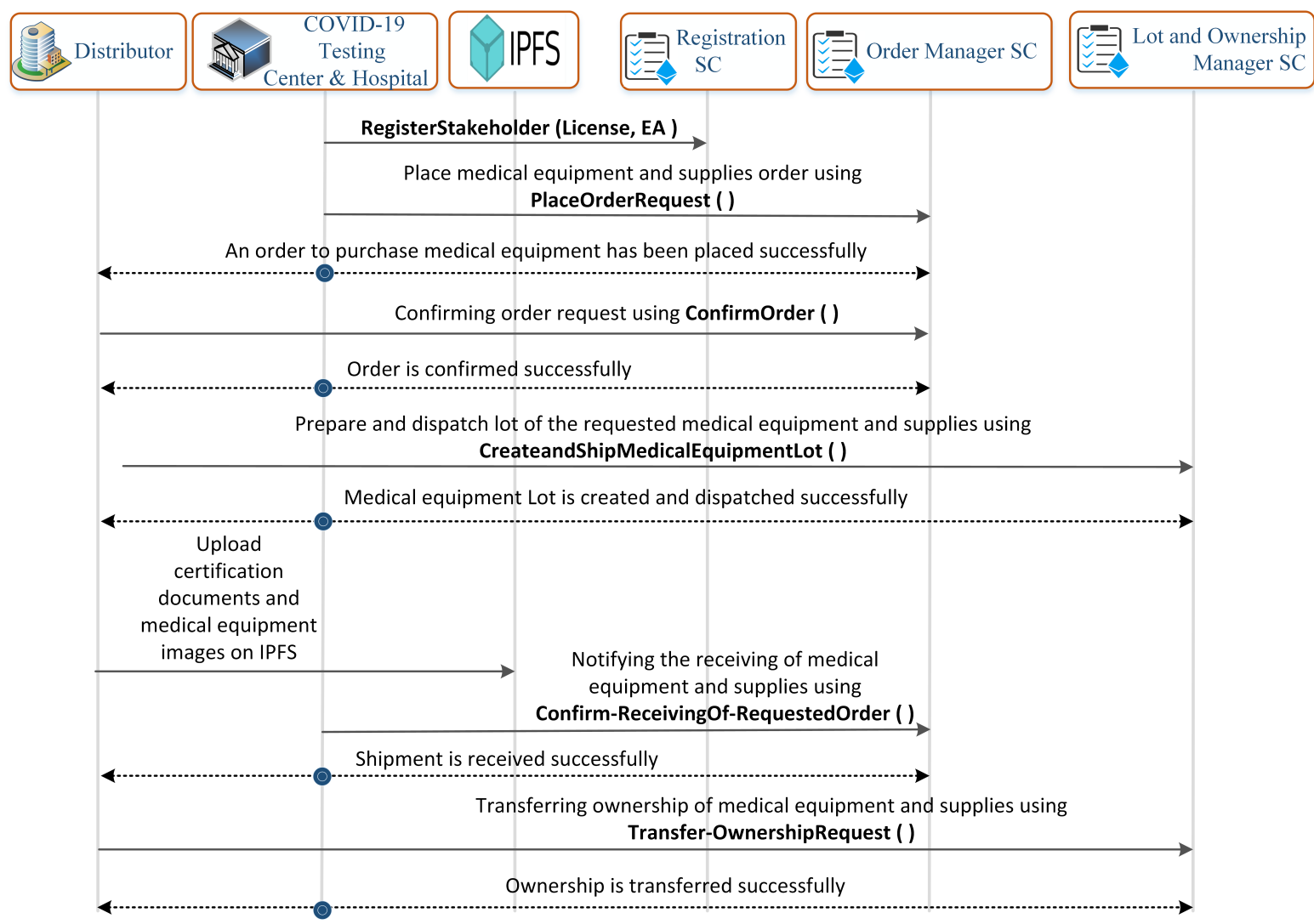

Figure 4: Sequence diagram showing the function calls and events between distributor and COVID-19 hospital \& testing center.

their role in the forward supply chain of COVID-19 waste delivery. It also assures that stakeholders perform actions in compliance with defined rules and regulations. Moreover, it imposes penalties on the stakeholder when an action that is non-complying with the stated rules is noticed. For instance, it can suspend the license of a participant such as a shipper if the COVID-19 waste is not properly handled during its disposal or shipment.

Distributed Storage: The participants of the medical waste management framework can store large-sized files on the distributed storage systems, such as IPFS, to overcome the scalability issues of blockchain [30]. In our proposed system, large-sized files related to COVID-19 medical equipment and supplies management include medical equipment certification and specification files. These files are stored on the IPFS and the hash of such files is stored on the blockchain ledger. The immutability feature of blockchain enables the users to verify the correctness of stored documents on the distributed storage systems.

Smart Contracts: Smart contracts replace the intermediaries and assure the governance of rules as agreed upon by the participating entities [31]. In this paper, we implement four major smart contracts; namely, order management smart contract, the lot and ownership management smart contract, Waste shipment handler smart contract, and registration smart contract. All these smart contracts constitute a set of functions that are called when a certain event happens in the COVID-19 medical equipment supply chain.

\section{B. Sequence of Operations}

This subsection explains sequence diagrams showing the function calls and blockchain-based events to automate the processes involved in the traceability of COVID-19 related medical equipment and their associated waste disposal.

The sequence diagram shown in Fig. 3 highlights the user's interaction with the system. All the participants are registered on the blockchain using a registration smart contract which is stored on the blockchain. The successful registration gives a unique identifier to the stakeholder which needs to be used in future for referring to the user or the services which are associated with it. After successfully registering a user on the blockchain platform, the distributor calls a function to deploy the order manager smart contract. The order manager smart contract is responsible for assisting the caller to place, confirm, or reject an order related to COVID-19 medical equipment and supplies. After a successful deployment of the order manager smart contract, the distributor stakeholder calls PalceOrderRequest function that as a result successfully places an order for the manufacturer to arrange medical equipment and supplies such as COVID-19 swab sticks. As a response, the manufacturer calls ConfirmOrder function to accept/reject the medical equipment order request by the distributor after analyzing the available medical equipment and supplies stock. Manufacturer prepares a lot of medical equipment and supplies and stores the details such as lot ID, quantity, name, type, certification number, and quality of the equipment on the blockchain platform. It also uses the IPFS system to store 


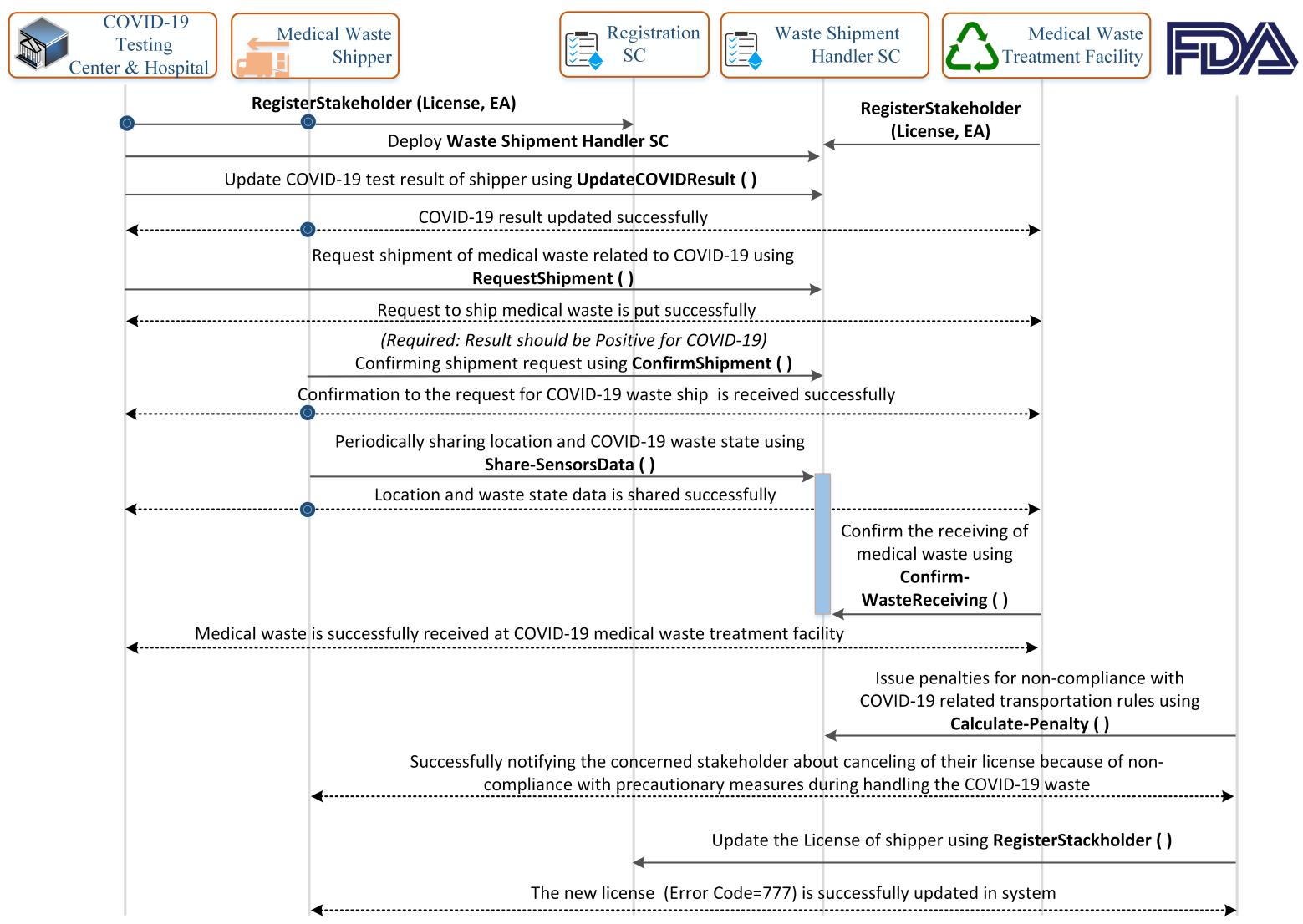

Figure 5: Sequence diagram showing the function calls and events among COVID-19 hospital \& testing center, medical waste shipper, FDA, and medical waste treatment facility.

large-sized medical equipment certification documents and images of the shipping packages. It stores the hash of these documents on the blockchain to assure the authenticity of medical equipment. On successfully receiving the medical equipment lot, the distributor notifies the manufacturer by calling Confirm-ReceivingOf-RequestedOrder function. Finally, the distributor is promoted as the new owner of the medical equipment lot by the manufacturer.

Fig. 4 captures the interaction between COVID-19 testing center/hospital and medical equipment distributor. Each entity in these systems is registered and represented by a unique Ethereum address. The call to PlaceOrderRequest function in order manager smart contract by the COVID-19 testing center places an order to the distributor to prepare and ship the medical equipment. In response, the distributor calls ConfirmOrder function to notify the COVID-19 testing center about the availability of requested medical equipment. An event is triggered by the smart contract to notify all related stakeholders about the response of the distributor. The distributor entity creates the lot and assigns new labels to the existing packages to ship it to the COVID-19 testing center. It also triggers Transfer-OwnershipRequest function to make COVID-19 testing center a new owner of the medical equipment lot. In contrast to Fig. 3 and Fig. 4, Fig. 5 captures the interaction between those stakeholders that control and manage COVID-19 equipment related to medical waste. The
COVID-19 testing center calls ShipmentRequest function to place a request to the certified shippers to ship the medical waste to the medical waste treatment facility. The shipper, in response, confirms its availability by calling ConfirmShipment function. Also, the shipment management smart contract notifies all the related entities about the response of the shipper. To comply with precautionary measures taken to mitigate the spread of COVID-19, waste bags are required to not be opened during its shipment. The shipper continuously stores state and location data on the edge server. In response, it calls ShareSensorsData function to store the location and state of the medical bags on the blockchain. The state of the waste bag indicates whether the bag was opened during the shipment or not. The smart contract notifies the relevant entities such as the medical waste treatment facility and COVID-19 testing center about the updated data to track the location and state of the medical waste during its shipment. This data is used by the FDA to verify that the COVID-19 medical waste was handled properly during its transportation to the medical waste treatment facility to minimize the spread of COVID-19. It cancels the license of the shipper if the COVID-19 medical waste is not handled properly.

\section{IMPLEMENTATION DETAILS}

This section presents the implementation details of the proposed blockchain-based approach. We use an Ethereum 

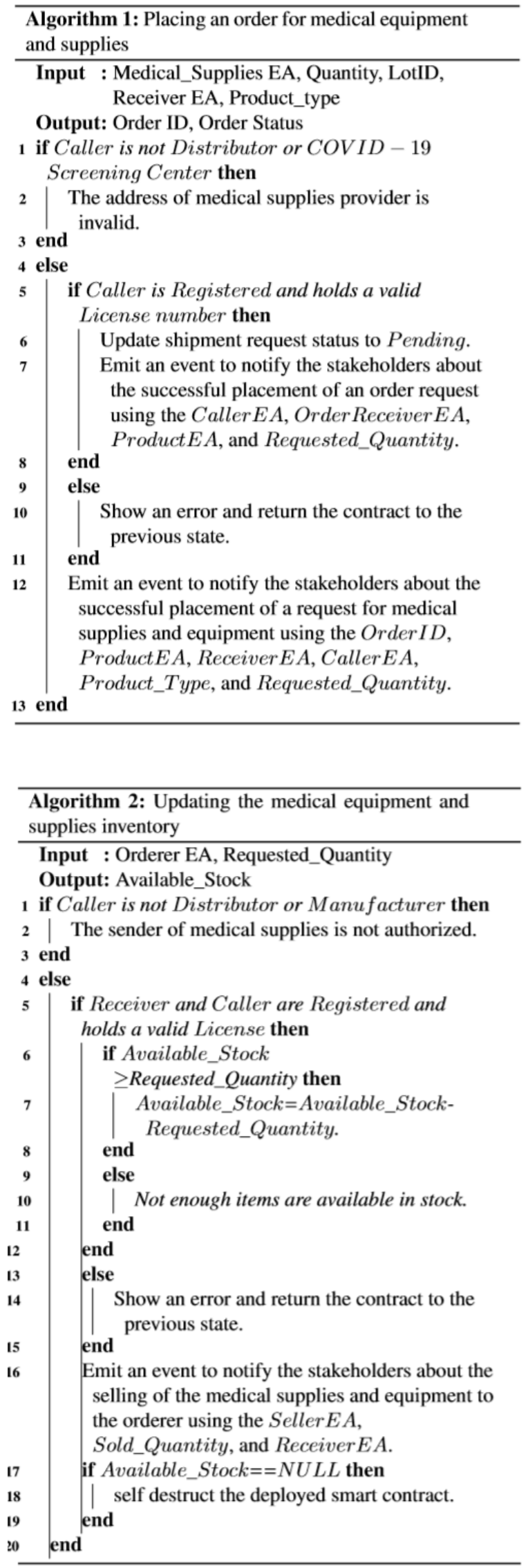

platform to implement smart contracts to govern the business activities of stakeholders. We use Remix IDE to develop, deploy, and test the smart contracts. The smart contracts developed to digitize the services of participating stakeholders include registration, lot and ownership management, order management, and shipment management.

Algorithm 1 presents a procedure to place an order request to the provider of COVID-19 related medical supplies and equipment. It requires the caller to provide input data such as the Ethereum address of the medical supplies and equipment providers, medical supplies quantity required, the Ethereum address of each requested medical equipment, and their type to successfully initiate the request and notify the participating stakeholders. It assures that only distributor or COVID-19 screening centers/hospitals can initiate a request for the medical supplies and equipment. It rejects any request for the medical supplies if initiated by any unregistered and unlicensed distributor/COVID-19 screening centers/hospitals. It generates an order identifier and notifies all the participating stakeholders about the pending request by emitting an event about registering of the recent order placement request.

The pending medical supplies and equipment requests are accepted or rejected by the distributor/manufacturer. Algorithm 2 highlights the business flow along with functionalities performed in selling the medical supplies to the orderer. It assures that only registered distributors and manufacturers with a valid license number can sell the medical supplies to the orderer for minimizing the fake drugs supplying from the unlicensed manufacturer or distributor. The available stock is verified to assure that the requested quantity of the medical supplies can be provided to the orderer. After preparing the lot for shipment to the orderer, the remaining medical supplies and equipment stock is updated accordingly. The participating stakeholders are notified about the sold medical equipment quantity and recipient of the order by emitting an event.

Medical equipment is used for screening, swab checking, and providing treatment to the people suffering from COVID19. The medical waste is generated once such medical supplies or equipment (e.g., COVID-19 swab sticks) are used in hospitals. This medical waste requires an immediate service to ship it to the medical waste treatment centers for disinfection and disposal. Algorithm 3 highlights the procedure along with system functions and $\log$ s to place a medical waste shipment request. This algorithm can only be triggered by the COVID19 testing centers/hospitals as shown in step 1 of Algorithm 3. It assures that the potential shippers of the COVID-19 medical waste should be registered, they have a valid license and recently tested negative for COVID-19 to further minimize the chances of COVID-19 spreading. A shipment identifier is created along with a pending shipping status to track the shipment request. Finally, it notifies the related stakeholders about the details related to this transaction by emitting an event.

The shipper confirms the shipment request of the COVID-19 screening center or hospital and starts the shipment. Algorithm 4 highlights the actions performed by the shipper during transportation of the waste related to COVID-19 medical supplies and equipment. Before data uploading, the system verifies 


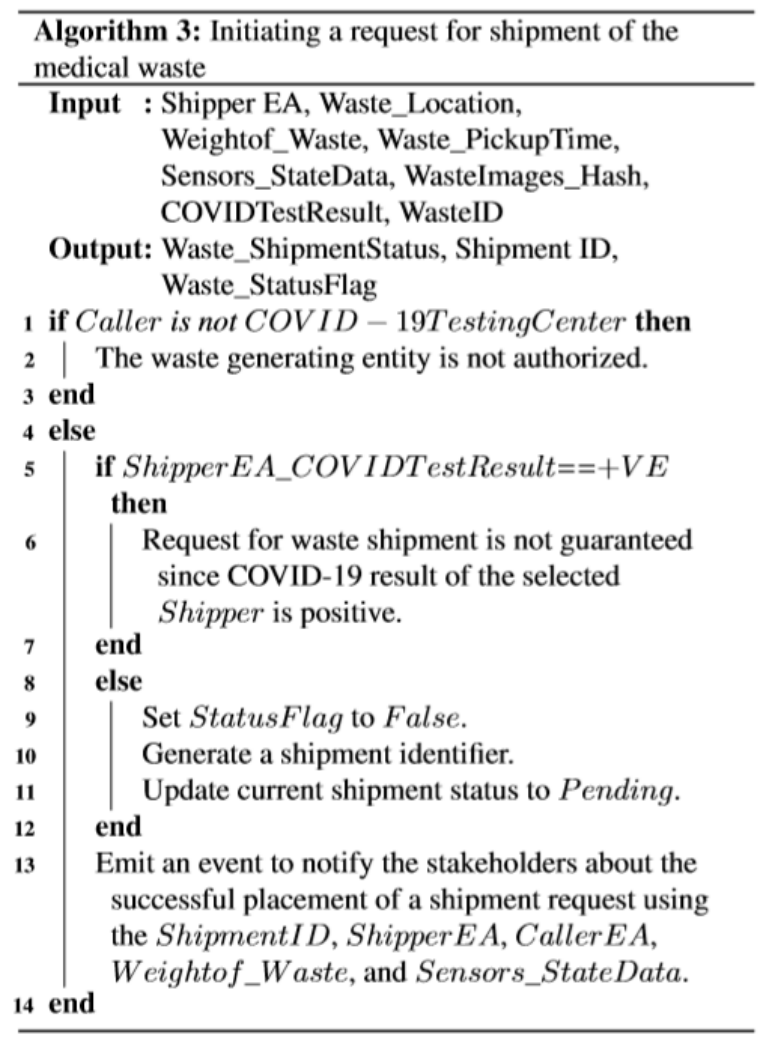

that the current status of the shipment is accepted. After this verification process, it allows the shipper to periodically upload the shipment location and waste container's state on to the chain until the waste reaches the medical waste treatment unit. It updates the shipment status to shipping to notify the related stakeholders about the shipment. On reaching the terminal of the waste treatment unit, it updates the status of the shipment to "reached" to inform the participating entities. It stores the logs of the events about the location and status of the shipment.

The rules to overcome COVID-19 from spreading have already been formulated to provide guidelines to the business personnel. Strict compliance with such rules by issuing penalties to the business stakeholders can significantly minimize the COVID-19 from spreading. Algorithm 4 successfully recorded the state of waste during its handling on the blockchain. Algorithm 5 analyzes the data related to the state of the waste during its handling and suggests penalties as well. It also proposes the FDA/Owner to invalidate the license of the relevant stakeholder. It calculates the violation count and issues the penalties to the shipper if the violation count is greater than the threshold. Also, it verifies and issues a penalty to the user if the weight difference between shipped and received waste is different.

\section{System Testing And VAlidation}

In this section, we present system testing and validation details. We explain all the functions and their outputs when they are executed in the Remix IDE environment.

The proposed blockchain-based system is developed and rigorously tested to verify that the rules implemented in
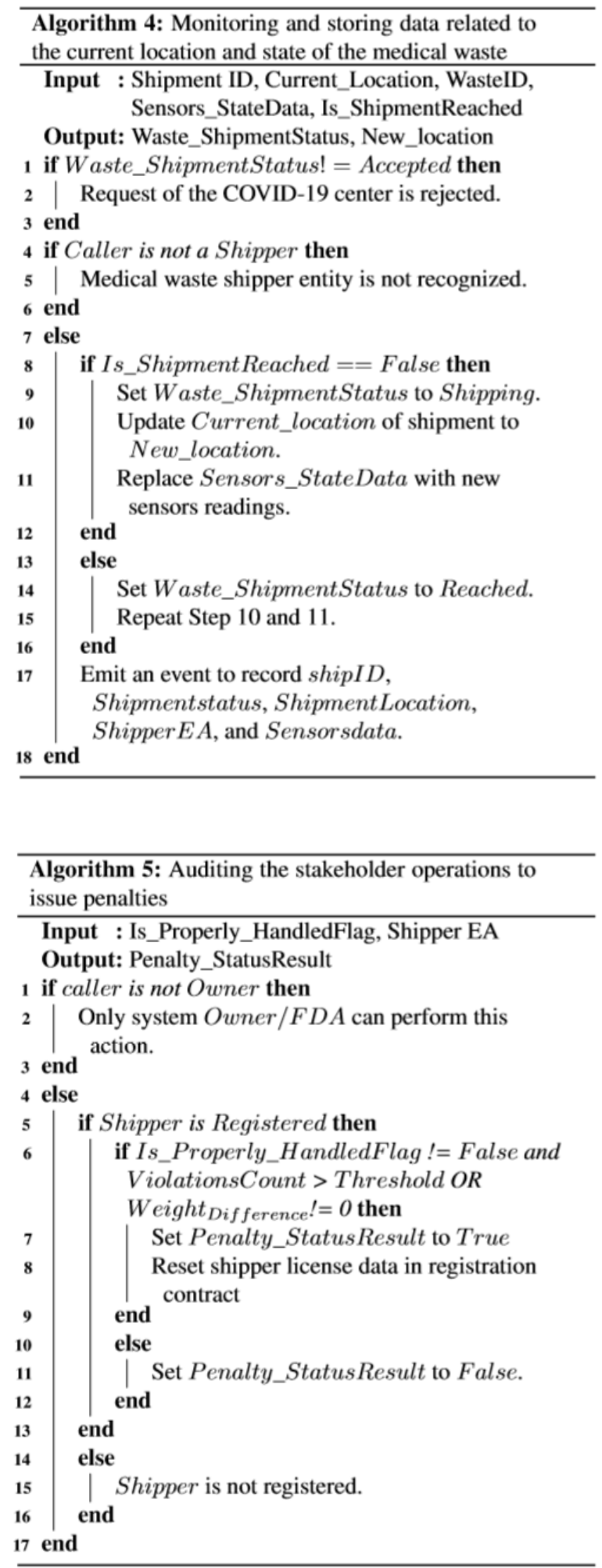

smart contracts are not violated during the business processes execution among system users. More specifically, it validates the behavior of functions implemented in smart contracts based on a set of inputs and scenarios. The modifiers are used for the function to assure that only authorized users can call a particular function. It generates an error when it recognizes unauthorized access to call a function. Many 
Table I: Ethereum addresses for participants

\begin{tabular}{ll}
\hline \hline Stakeholder Name & Ethereum Address \\
\hline Medical Equipment Manufacturer & 0x78731D3Ca6b7E34aC0F824c42a7cC18A495cabaB \\
Distributor & 0x4B20993Bc481177ec7E8571ceCaE8A9e22C02db \\
COVID-19 Testing Center & 0xAb8483F64d9C6d1EcF9b849Ae677dD3315835cb2 \\
Medical Waste Shipper & 0x617F2E2fD72FD9D5503197092aC168c91465E7f2 \\
Medical Waste Treatment Facility & 0x17F6AD8Ef982297579C203069C1DbfFE4348c372 \\
FDA & 0x5B38Da6a701c568545dCfcB03FcB875f56beddC4 \\
\hline
\end{tabular}

events are implemented in smart contracts that transparently record the logs of the transactions when they are executed. Finally, the entities of the system including smart contracts, system users, and medical equipment and supplies are uniquely identified using their Ethereum addresses. This system can be used to trace the medical equipment and supplies from their manufacturing till the waste disposing stage. The use of penalties for breaching the rules during COVID-19 waste handling can significantly control the spread of COVID-19.

Each entity in the system is represented by a unique Ethereum address. Table I highlights the Ethereum addresses of the participating stakeholders. We have assigned Ethereum addresses to each medical equipment or supplies to uniquely identify it. But, the barcodes or QR-codes stamped on the medical equipment or supplies can be registered on the blockchain to track such equipment or supplies.

Fig. 6 shows the results on executing the PlaceOrderRequest function of the OrderManager smart contract in remix IDE. PlaceOrderRequest function enables the participants such as medical equipment distributor and COVID-19 testing center to register a request for system participants responsible to arrange and supply medical supplies and equipment such as COVID-19 swab testing kit. In the presented result in Fig. 6 , PlaceOrderRequest function is triggered by the distributor stakeholder of the proposed system. The system validates the identity of the distributor to verify that it is a registered user and holds a valid license number. Since the distributor gets the medical supplies from the manufacturer, hence system verifies that the receiver of this function call should be the manufacturer stakeholder. On successful execution of this function, an event named Orderplaced is emitted that stores the transaction log on the blockchain. The transaction log includes medical equipment EA, OrderID, Equipment Name, requested LotSize, and address of the order receiver.

On successfully executing the PlaceOrderRequest function, the system sets the status of the transaction to pending, thereby enabling the users to view the current status of the order for medical equipment. The receiver of the order can examine the needs and requirements of the order request (in offline mode), and it notifies the orderer about its response using on-chain resources. Fig. 7 shows the result in executing the ConfirmOrder function of the OrderManager smart contract. The manufacture uses this function to publish its response by either accepting or rejecting the distributor request. This function is triggered by the manufacturer stakeholder only since the orderer entity in Fig. 6 is a distributor. On successful execution of the ConfirmOrder function, the manufacturer response is recorded on the blockchain by emitting an event called OrderReceived. This event stores the updated status of the order along with the OrderID that was generated by the PlaceOrderRequest function. The presented result in Fig. 7 shows that the medical supplies request transaction was successfully accepted by the manufacturer.

After accepting the order request, the manufacturer updates the record of medical supplies inventory based on the lot size requested by the distributor. In the orderManager smart contract, the UpdateInventory function is responsible to assure that the stock available in the store can fulfill the requirements of the distributor. Further, it updates the remaining medical supplies stock by subtracting the requested quantity from the available stock. On successful execution, as can be seen in Fig. 8, it emits equipmentSoldtoOrderer event to record the logs on blockchain to show that medical supplies are sold out to the distributor. It records the address of the distributor, quantity sold, available quantity, Ethereum address of the medical supplies, and the time when this action is performed. After updating the inventory, the ownership is transferred and the distributor is updated as the new owner. Fig. 9 shows the result of executing the TransferOwnership function of the orderManager smart contract. The system verifies that only the shipper of the medical supplies can call the TransferOwnership function. In our case, this function was called by the manufacturer. On successful execution of this function, an event is emitted and it stored the type and Ethereum address of the new owner, e.g., distributor.

Fig. 10 shows the results on executing the ConfirmReceived function of the OrderManager smart contract in remix IDE. This function requires that the caller of this function must be the orderer entity. Also, it requires that the shipment order status must be previously set as accepted prior to ConfirmReceived function execution. On successful execution of this function, an event called OrderReceived is emitted that logs the OrderID.

Fig. 11 and Fig. 12 refers to a scenario wherein the order for medical equipment and supplies is placed by the COVID19 testing center. Fig. 11 shows the result of executing the PlaceOrderRequest function in RemixIDE. In contrast to Fig. 6 , the originator of the transaction in Fig. 11 (See "from" part) is the COVID-19 testing center. After successful execution of this function, an event is generated that records the transaction log by immutably storing the Ethereum address of the orderer, medical equipment, and receiver, Lot size, and orderID to uniquely identify the order. Fig. 12 shows the result when the ConfirmOrder function is called by the medical supplies distributor. The address of the caller of this function (i.e., distributor) can be seen in the "from" section in Fig. 12. 


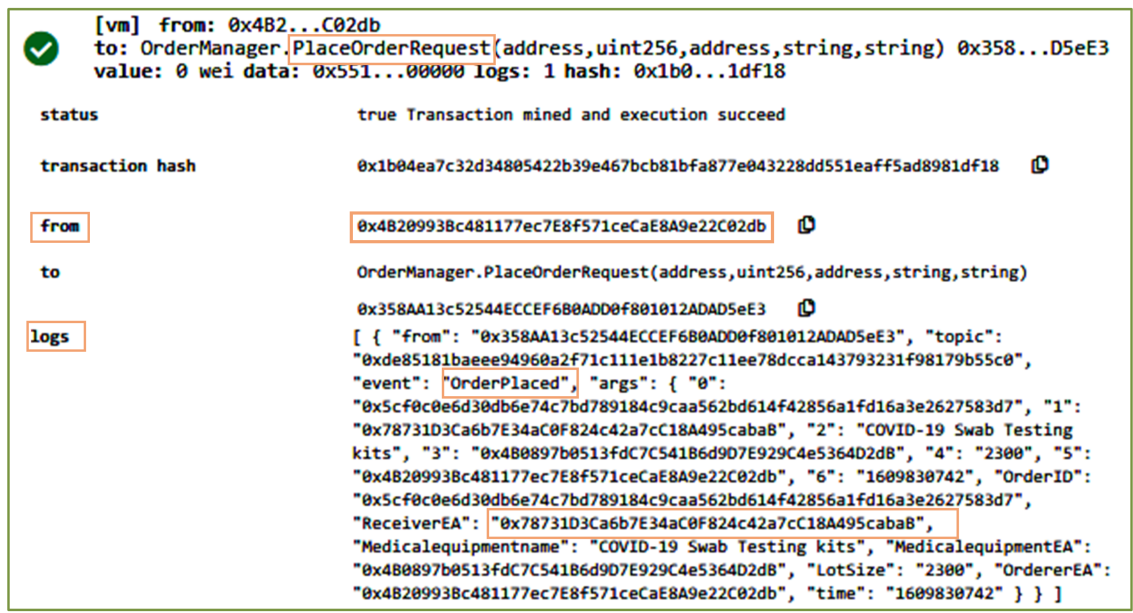

Figure 6: Logs showing the successful order placement for medical supplies and equipment.

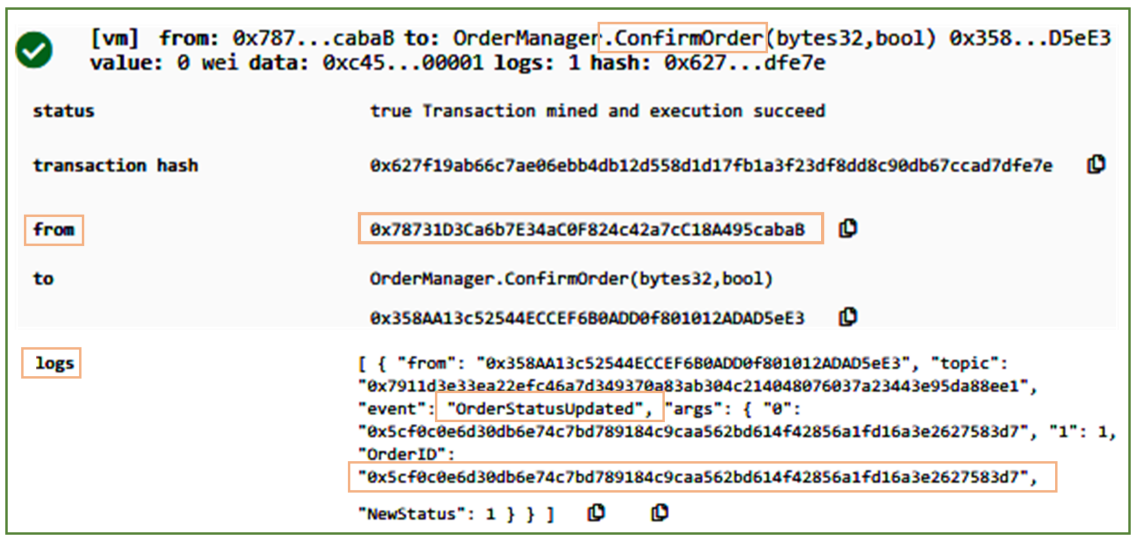

Figure 7: Logs showing the order confirmation for medical equipment and supplies.

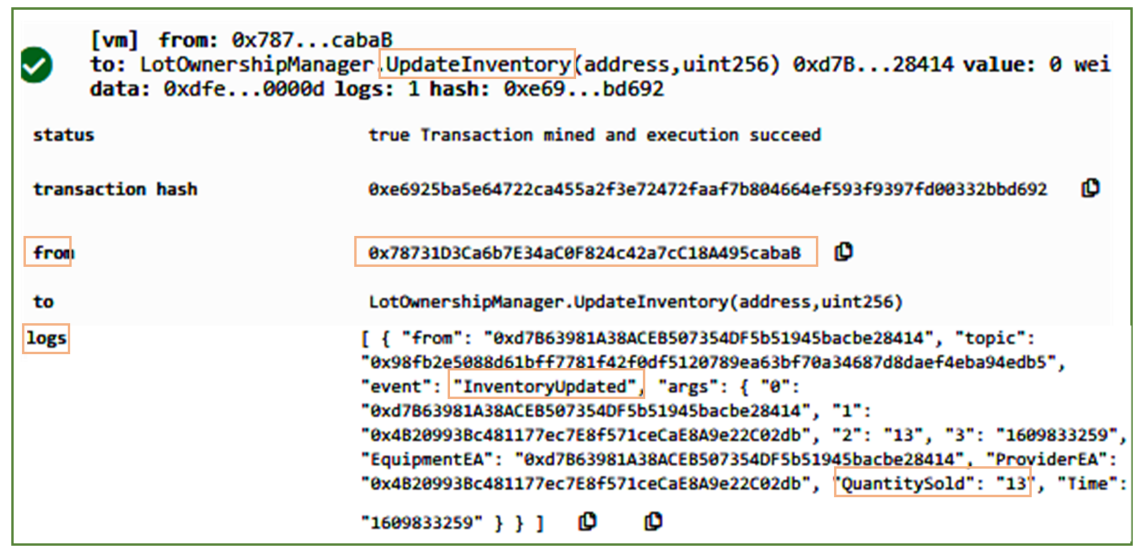

Figure 8: Logs showing the inventory updating about COVID-19 related medical equipment and supplies.

Fig. 13 shows the result generated in Remix IDE when the UpdateInventory function of the Ordermanager smart contract is called. On successful execution, the log of the transaction is generated and it transparently stores COVID-19 testing center as the receiver of the order in addition to many other important parameters such as quantity sold and remaining quantity, and the orderID. On successful execution of TransferOwnerhip function, the Ethereum address of the new owner of medical supplies is immutably recorded on the blockchain as can be seen in Fig. 14.

Medical equipment and supplies are used by the medical staff in COVID-19 testing centers or hospitals to test and provide treatment to COVID-19 patients. Many medical equipment and supplies such as personal protective equipment 


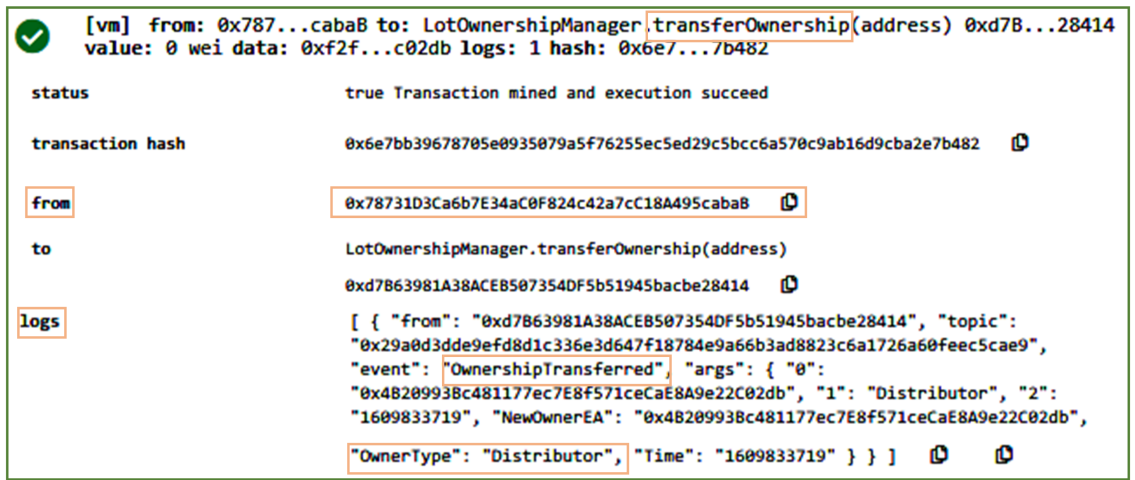

Figure 9: Logs showing the transferring of ownership to the distributor.

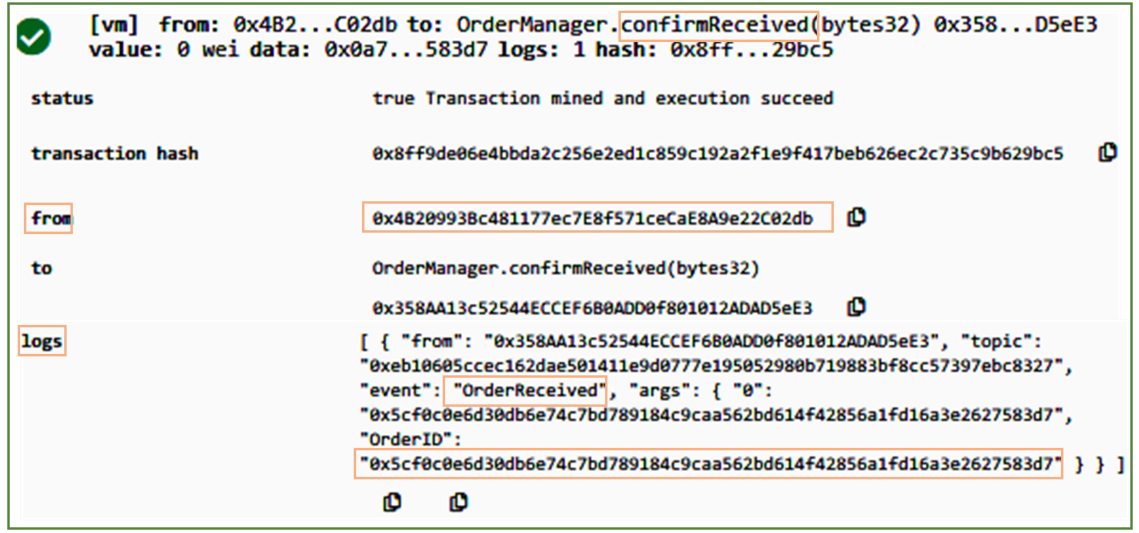

Figure 10: Logs showing the confirmation of receiving of the order by the distributor.

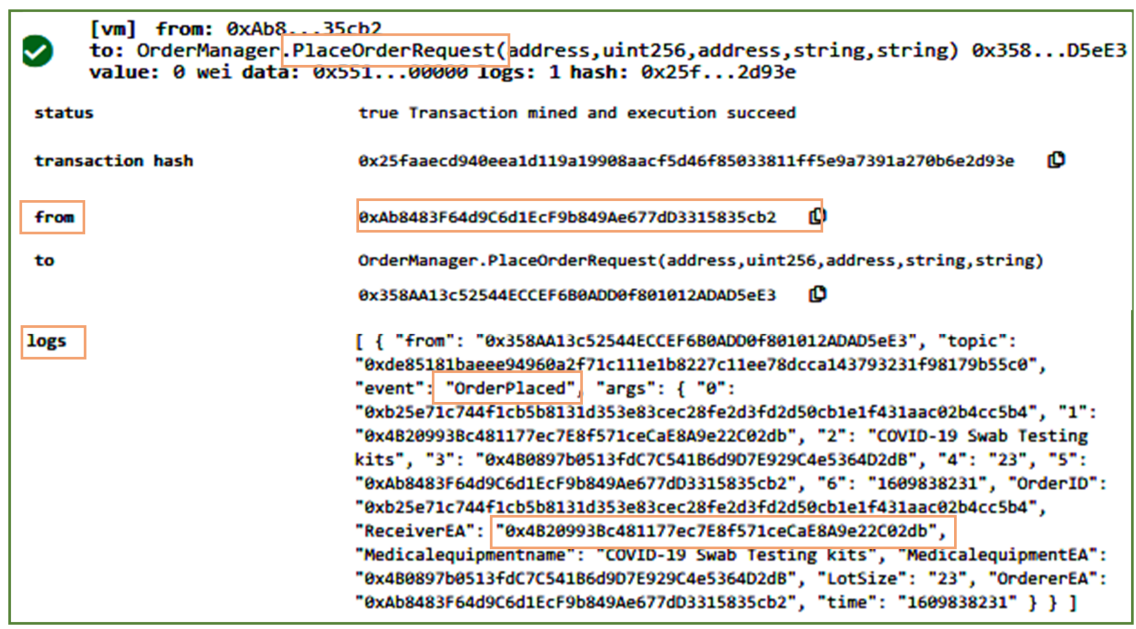

Figure 11: Logs showing medical equipment and supplies order placement by the COVID-19 testing center.

and swab testing tubes, are specially designed to be used for one time only. Thus, these equipment require immediate disposal after their usage. Considering the contagious nature of coronavirus, the COVID-19 related medical waste demands to be carefully handled by the stakeholders during its temporary storage in the hospital's medical waste storage room, waste shipment, segregation, disinfection, and disposal at the medical waste treatment facility. Thus, the handler of the COVID-19 related waste should not have COVID-19 symptoms that can further assist in minimizing the chances of spreading COVID19.

Our proposed system has implemented a shipment handling smart contract to assure that the medical waste shipper should have a valid COVID-19 result to ship the medical waste. Fig. 15 highlights an error generated by the system because selecting a medical waste shipper who does not have a valid 


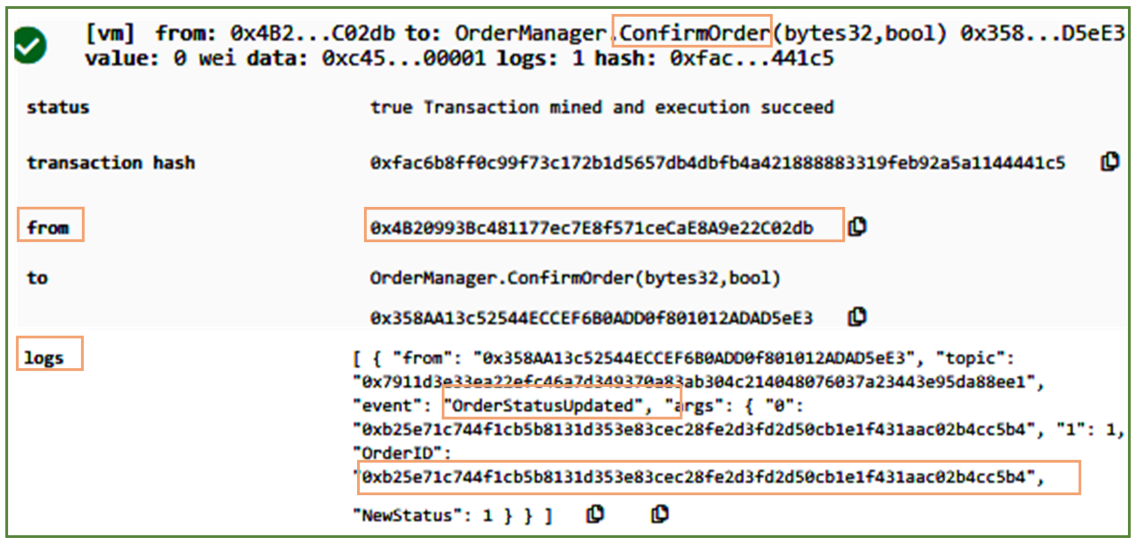

Figure 12: Logs showing the order confirmation by the distributor.

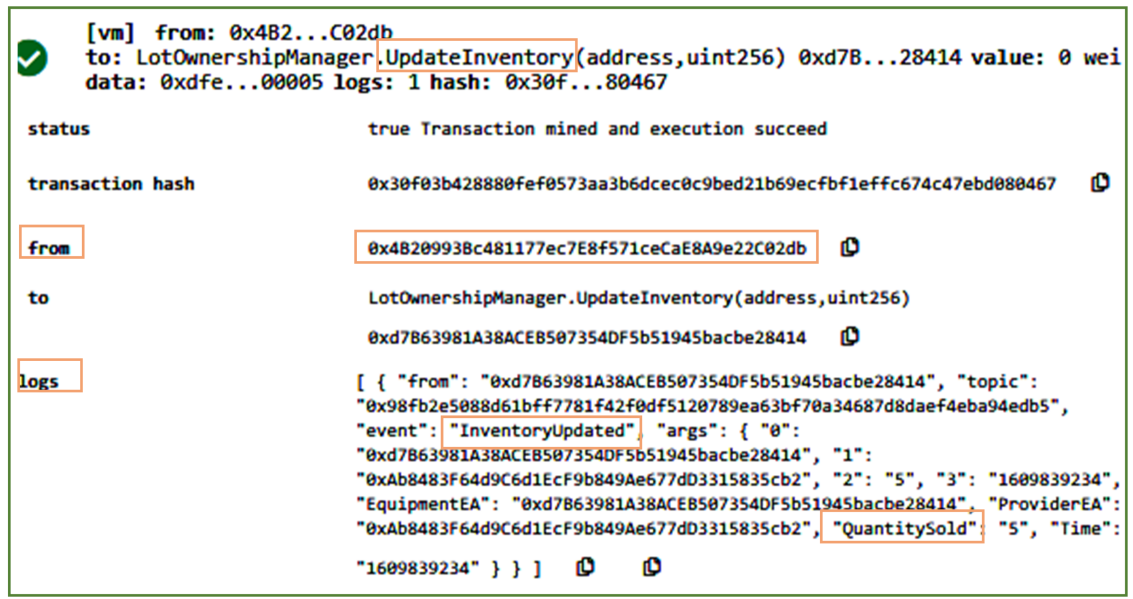

Figure 13: Logs showing the inventory updating of the medical equipment and supplies by distributor.

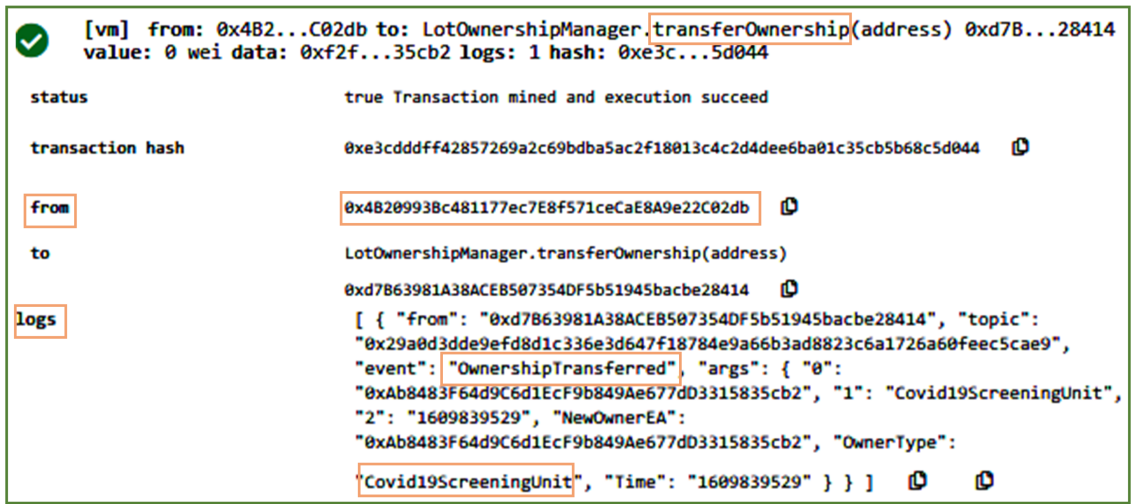

Figure 14: Logs showing the ownership transferring to the COVID-19 testing center.

COVID-19 result is not permitted. This error has occurred in response to the hospital manager's interaction with the PlaceShipmentRequest function.

Fig. 16 shows the result on calling a PlaceShipmentRequest function by the COVID-19 testing center. This function accepts the shipper's Ethereum address along with the weight of COVID-19's waste, earliest pick-up time, sensors data attached to the waste bags, and the hashes of the waste certificates and 3D images. It proceeds further only if the shipper has a valid COVID-19 result. It sets the transaction status to pending to notify the shipper that the shipment services for medical waste are required. On successfully executing this function, it emitted an event and records the Ethereum address of the shipper and waste generator, shipment ID, and other necessary details on the blockchain. The shipment request is examined by the shipper to either accept or reject it based on the function's 


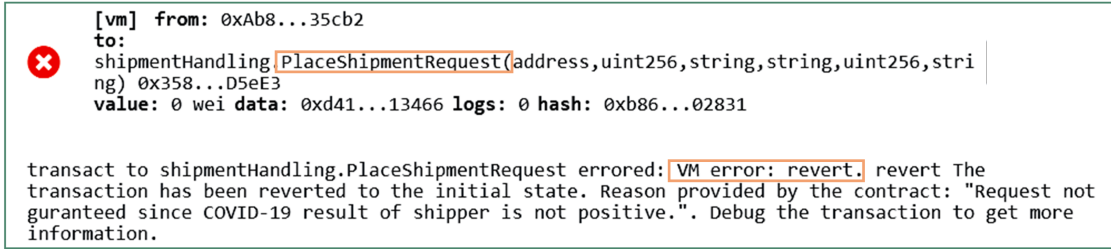

Figure 15: Error highlighting the user action's non-compliance with waste handling rules.

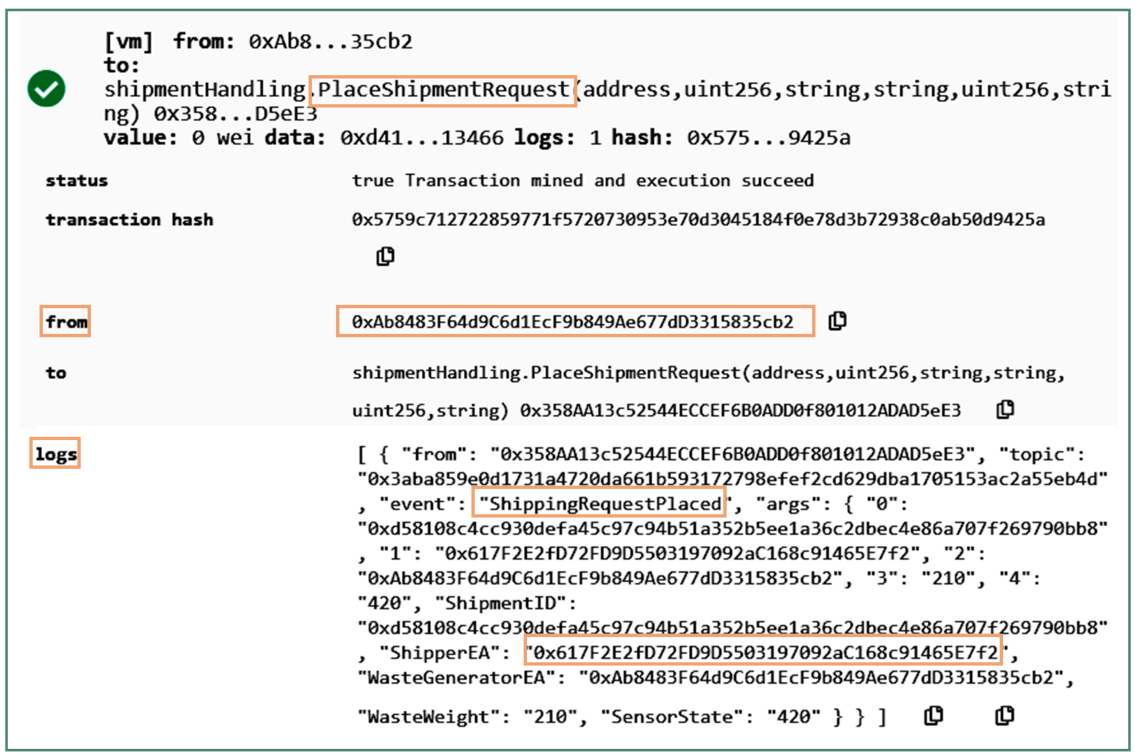

Figure 16: Logs showing the placement of request to ship COVID-19 medical waste.

input parameters. After accepting the medical waste shipment request, the shipper collects the waste and routes it to the medical waste treatment facility.

Fig. 17 shows the result on calling a UpdateShipmentStatus function. This function can be called only if the medical waste shipper has already accepted the waste shipment request. It continuously records the updated data (company's local server) related to the current location, state, and status of the shipment. This data is periodically stored on the blockchain. It emits the event called shipmentstatusandlocationupdated to record the current location and state of sensors to notify the users about the current location and state of the shipment. The state of the shipment enables the users to know that at which time and place the sealed bags of COVID-19 waste were unsealed.

The COVID-19 related waste is usually packed in multiple boxes that are carefully sealed to minimize the chances of spreading COVID-19 during its shipment stage. Many sensors are mounted inside such boxes to identify any human attempt made to open and stealing them (if any). The deployed sensors can sense, monitor, and record this data on to the blockchain ledger. Fig. 18 shows the results on calling the PenaltyCalculationResult function. This function can be called by the owner of the smart contracts. As can be seen in Fig. 18 , no penalty is issued to the medical waste shipper since no non-compliance with rules for COVID-19 waste handling is identified. On successful execution of this function, an event has been emitted that can store whether the license of the shipper is canceled or not based on the violations count.

In contrast to Fig. 18, Fig. 19 presents the results in which a penalty in terms of cancellation of license of a stakeholder has been issued to the COVID-19 medical waste shipper. Once the shipper's license is canceled, he/she cannot further participate in the shipping services. Fig. 20 shows graphically the results on executing the PenaltyCalculationResult function. It highlights that the license of the current shipper has been invalidated. It also shows that 4 violations are reported during the medical waste shipment.

\section{Discussion}

This section presents the cost and security analysis of the proposed solution. It also outlines the future research challenges.

\section{A. Cost Analysis}

The Ethereum blockchain platform meters the transaction computations in the unit of gas. The user is required to set a maximum amount of gas for each transaction to execute it. Each Ethereum virtual machine (EVM) operation consumes a certain amount of gas. Also, in smart contracts, storage variables, loops, nested decision making, mappings, and manipulations share the major portion of gas consumption. During transaction execution, if the required amount 


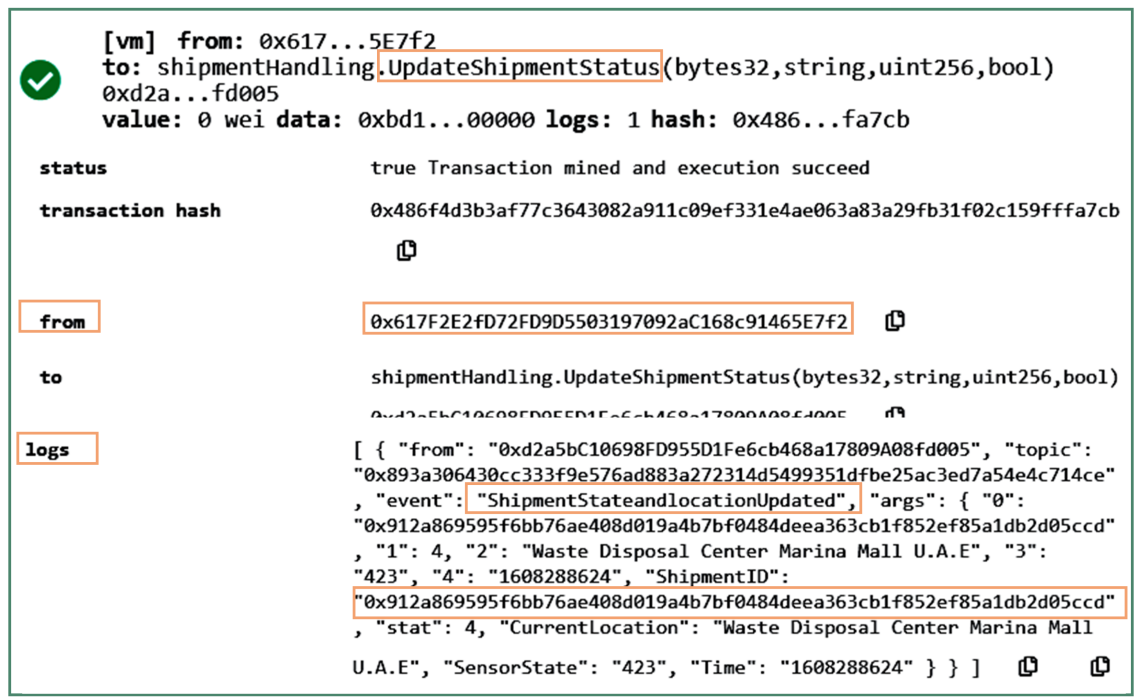

Figure 17: Logs showing the updating of location and state of the medical waste during its shipment.

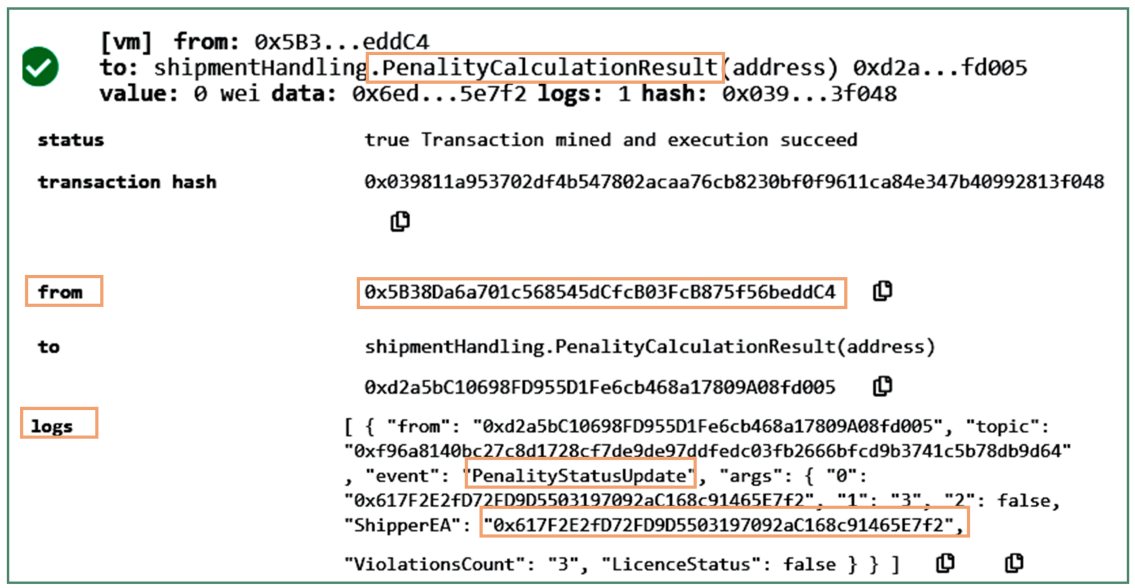

Figure 18: Logs showing the successful and rules-based shipment of medical waste.

\begin{tabular}{|c|c|}
\hline $\begin{array}{l}{[\mathrm{vm}] \text { from }} \\
\text { to: shipm } \\
\text { value: } \theta\end{array}$ & $\begin{array}{l}\text {.eddc4 } \\
\text { 0x.PenalitycalculationResult (address) oxb27....07c2c } \\
\text { true Transaction mined and execution succeed } \\
\text { 0x6933fae791f8e5d195741b97346cfdcbdbe日b7d66c4be93006333945a08bf2b1 } \\
\text { (t) }\end{array}$ \\
\hline from & Ox5B38Da6a701c568545dCfcB03FcB875f56beddC4 \\
\hline \multirow[t]{2}{*}{ to } & shipmentHandling.PenalityCalculationResult(address) \\
\hline & 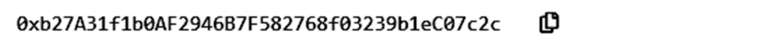 \\
\hline \multirow[t]{2}{*}{ logs } & 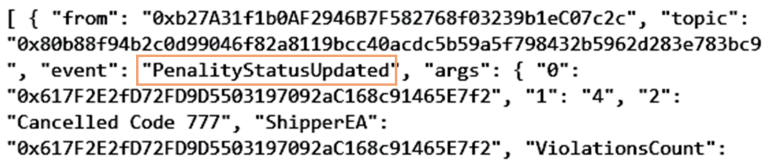 \\
\hline & 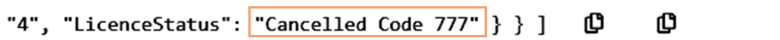 \\
\hline
\end{tabular}

Figure 19: Logs showing the non-compliance with COVID-19 waste handling rules and shipper's license cancellation.

of gas is higher than the set Gas-limits then EVM halts the operations and charges the users to make sure that non- terminating computations cannot be performed to slow down the network. Because of the high flexibility, Ethereum allows 
Table II: Gas cost in Ether of the functions in smart contracts.

\begin{tabular}{lllllll}
\hline \hline $\begin{array}{l}\text { Function } \\
\text { Name }\end{array}$ & $\begin{array}{l}\text { Transaction } \\
\text { Gas }\end{array}$ & $\begin{array}{l}\text { Execution } \\
\text { Gas }\end{array}$ & $\begin{array}{l}\text { Fastest Execution } \\
\text { (Ether) }\end{array}$ & $\begin{array}{l}\text { Avg. Execution } \\
\text { (Ether) }\end{array}$ & $\begin{array}{l}\text { Slow Execution } \\
\text { (Ether) }\end{array}$ & $\begin{array}{l}\text { Transaction Cost } \\
\text { (USD) }\end{array}$ \\
\hline RegisterTestingCenter & 65893 & 43021 & 0.0038218 & 0.0029652 & 0.0025039 & 0.441763077 \\
RegisterDistributor & 65927 & 43055 & 0.0038238 & 0.0029667 & 0.0025052 & 0.441992436 \\
WasteShiperExists & 23900 & 1220 & 0.0013862 & 0.0010755 & 0.0009082 & 0.160233726 \\
DistributorlicenseisValid & 24878 & 2198 & 0.0014429 & 0.0011195 & 0.0009454 & 0.166796922 \\
PlaceOrderRequest & 222507 & 196691 & 0.0189131 & 0.015353 & 0.0106803 & 1.884325329 \\
GetOrderID & 22293 & 1021 & 0.001293 & 0.0010032 & 0.0008471 & 0.149453853 \\
ConfirmOrder & 71979 & 48339 & 0.0041748 & 0.0032391 & 0.0027352 & 0.482571336 \\
GetOrderState & 24693 & 1245 & 0.0014322 & 0.0011112 & 0.0009383 & 0.165544269 \\
ConfirmReceived & 36082 & 12634 & 0.0020928 & 0.0016237 & 0.0013711 & 0.241903173 \\
UpdateInventory & 43466 & 20594 & 0.002521 & 0.001956 & 0.0016517 & 0.291409431 \\
TransferOwnership & 62136 & 39456 & 0.0036039 & 0.0027961 & 0.0023612 & 0.416586516 \\
UpdateHealthStatus & 51285 & 28477 & 0.0029745 & 0.0023078 & 0.0019488 & 0.343826784 \\
PlaceShipmentRequest & 181512 & 156464 & 0.0105277 & 0.008168 & 0.0068975 & 1.216925925 \\
GetShipmentID & 22339 & 1067 & 0.0012957 & 0.0010053 & 0.0008489 & 0.149771427 \\
ConfirmShipmentRequest & 62151 & 38511 & 0.0036048 & 0.0027968 & 0.0023617 & 0.416674731 \\
ConfirmShipmentReceiving & 40384 & 16936 & 0.0023423 & 0.0018173 & 0.0015346 & 0.270749478 \\
GetPenalty & 31353 & 8673 & 0.0018185 & 0.0014109 & 0.0011914 & 0.210198702 \\
UpdateShipmentStatus & 27881 & 3409 & 0.0016171 & 0.0012546 & 0.0010595 & 0.186927585 \\
GetWasteState & 24583 & 1135 & 0.0014258 & 0.0011062 & 0.0009342 & 0.164820906 \\
PenalityCalculationResult & 41538 & 18858 & 0.0024092 & 0.0018692 & 0.0015784 & 0.278477112
\end{tabular}

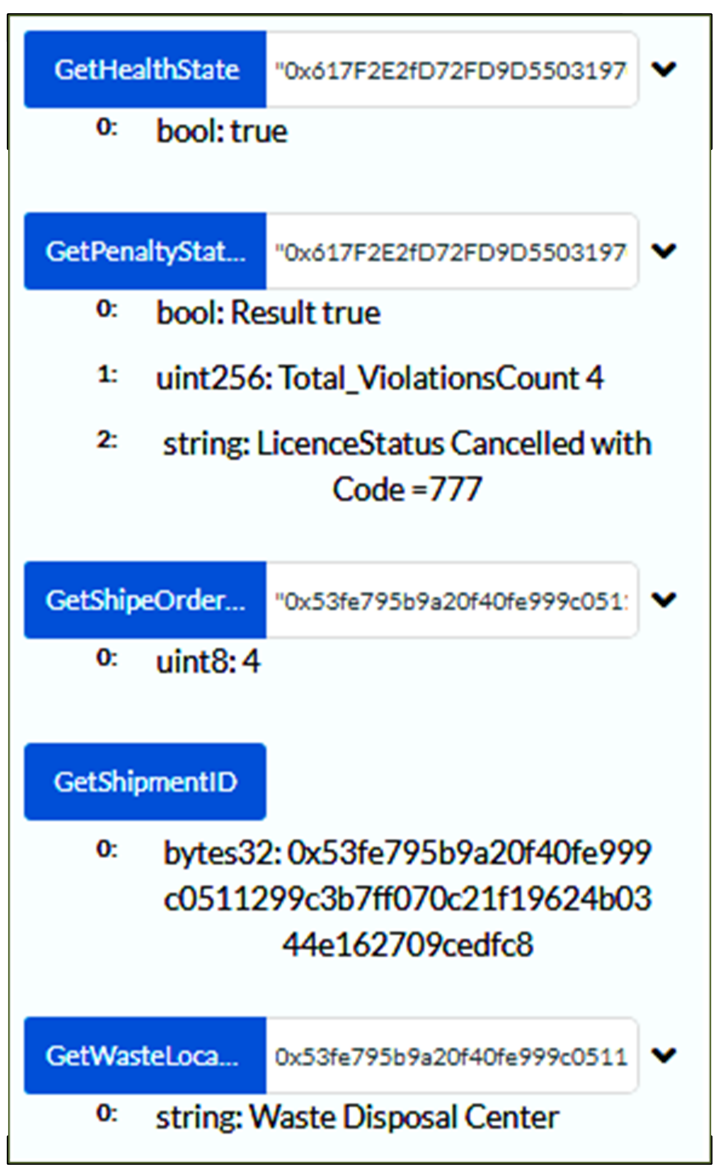

Figure 20: Highlighting the total violations count and license cancellation of shipper on calling GetPenaltyStatus function.

the users to specify the cost of gas consumption based on their requirements (slow or fast execution). Hence, users can offer more gas price for the miners to prioritize their transactions.
The Remix IDE calculates and stores the transaction and computation cost as part of the transaction log [32], [33]. The gas prices can be easily specified in Gwei. Gwei is the most known unit of ether. An analysis of a smart contract or method for gas consumption can be helpful to design lightweight and computationally inexpensive smart contracts.

Ethereum Gas Station estimates the gas price for the Ethereum-based transactions. It offers different speeds of executing the transactions that are largely affected by the gas price offered by the user [32], [34]. For example, it can offer a price for the given units of gas based on fastest, fast, average, and slow transactions execution speed. The gas price in Ethereum is variable since it changes over time and highly depends on the congestion in the network. Hence, the gas price could be different during different times of the day. The current research has used the gas prices offered by the ETH Gas station on 26 December 2020. At the time of writing of this paper, the transaction fee for 21000 gas units is observed 58, 45, and 38 Gwei for fastest, average, and slow transaction execution speeds. Moreover, to convert Ethers into USD, we used the conversion rate from May 2020 to represent a more stable and realistic value.

Table II shows both transaction and computations gas units consumed by functions of the proposed smart contracts when executed in Remix IDE. It also shows the transaction fee for the fastest, average, and slow transaction execution speeds for each function. The fastest transaction execution speed requires more Ethers than slow or average execution mode. Among all the specified functions in Table II, PlaceShipmentRequest and PlaceOrderRequest need the highest number of tokens for their successful execution since these functions have involved various complex operations. For instance, placeShipmentRequest function has implemented several structures, mappings, manipulations, and modifiers to assure the correct business operations among participating stakeholders. It also has implemented many modifiers to assure that, (1) the potential medical 
waste shipper has no COVID-19 symptoms, (2) the caller of the function is a business representative of COVID-19 testing hospital, and (3) the shipper has a registered license number. It also implements structures to record the details about the medical waste material and shipper. The GetOrderID function requires the least number of Ethers for its execution among all functions.

\section{B. Security Analysis}

Herein, we present a detailed security analysis of the proposed solution that offers high security, robustness, and resilience features. Below is given a brief discussion on the key security and privacy issues that are addressed in our system. It also discusses the security analysis of the implemented smart contracts.

Availability and Fault Tolerance: Blockchain technology has gained tremendous popularity in the healthcare sector because of its inherited security features. Unlike centralizedbased systems, the decentralized feature makes it highly acceptable and dependable to healthcare organizations. Furthermore, the decentralization makes the system highly faulttolerant and available despite the occurrence of malicious codes such as the denial of service (DoS) attacks. Therefore, such high availability makes it acceptable to the industries. Since our system has been implemented on the Ethereum blockchain platform, the services of our system will always remain available to the participating stakeholders.

Data Integrity and Immutability: Data manipulation is one of the serious threats to the integrity of the data. Among many, approaches such as append-only, data certification, and sophisticated access control can enable organizations to protect their data. In the blockchain, through cryptographic functions, data integrity is preserved. The hashing algorithms make the blockchain transactions immutable. In our proposed waste management system, the transactions of the participants such as the manufacturer and COVID-19 testing center are digitally signed before they are written on the blockchain. Thus, the immutability feature of blockchain assures that these transactions cannot be edited, modified, or deleted. Hence, the proposed solution preserves data integrity.

Non-repudiation and Digital Signatures: Digital signatures are based on mathematical schemes and are often used to verify the authenticity of a transaction. Also, digital signatures provide non-repudiation service to the existing systems, since the signer cannot deny that he/she did not sign the transaction. In the Ethereum blockchain platform, each transaction of the user is secured by the digital signature. For instance, a waste treatment facility might have sent a waste shipment confirmation transaction to the hospital. The blockchain assure that the transaction is signed with the private key of the waste treatment facility. This transaction cannot be modified or deleted because of immutable blockchain technology. At any time, this transaction cannot be denied by the waste treatment facility since the originator of the transaction can be verified by the signature.

Resilience against Man-in-middle Attacks: In a Man-inmiddle attack, a user intercepts communication between two parties to secretly eavesdrop or modify the exchanging data. Blockchain technology assures that no man-in-middle attack can be successfully launched. It requires the users to sign digitally the encrypted transactions to immutably store it on the blockchain platform. The launch of the Man-in-middle attack is successful only if the malicious actor has successfully retrieved the private key of the communicating parties. The miners of the Ethereum blockchain platform cannot approve those transactions wherein signatures do not match with the signatures of the legitimate users. Our system is comprised of several stakeholders who are often located in geographically different locations. Thus there is a high probability of Manin-middle attacks. Since our system is implemented on the Ethereum blockchain platform, hence it is safe against Manin-middle attacks.

Protection against Smart Contract Vulnerabilities: An ill-designed or poorly written smart contract can exhibit low performance, limited security, and high transaction execution fee. It is highly recommended to write smart contracts carefully to avoid any exploitation of smart contracts for malicious activities. There are many tools available that statically analyze the smart contracts (e.g., SmartCheck and Securify) and provide guidelines to improve the cost, performance, and security of smart contracts. The guidelines state that smart contracts should be checked for infinite loops, floating-point operations, reentrancy errors, and common bugs that can make the smart contract highly vulnerable to attacks. We improved our smart contracts by analyzing them using the SmartCheck [35] tool. Our smart contracts have shown no bugs and vulnerabilities such as infinite loops, race condition, integer division, Denial of Service (DoS) by an external, malicious external library, and locked transfers.

\section{Future Research Challenges}

In this subsection, we enumerate and discuss several important challenges remaining to be addressed.

GDPR and Data Privacy: Blockchain is a highly reliable technology as it efficiently protects the data against manipulation. It accomplishes security through transparency and storing data on multiple nodes. However, the transparency and distributed storage of data can greatly affect data privacy. The public blockchain platforms such as Ethereum face data privacy assurance challenges since all transactions, addresses, and data are publicly available on the ledger. Such data can be accessed to invade the privacy of user data. The EU General Data Protection Regulation (GDPR) laws protect the data related to the organizations that participate in business processes in the forward supply chain and waste management of medical equipment and supplies. Even though our study has used a registration smart contract and function modifiers to control data access on the chain. However, due to data transparency, many of the data can be accessed by others. Hence, compliance with GDPR laws on employing public blockchain is challenging. The non-compliance with GDPR can affect the adaptability of the proposed system.

Regulation: Blockchain technology offers many advantages to the stakeholders involved in the forward supply chain 
of medical equipment and their disposal in terms of lower transaction cost, streamlined operations, improved system efficiency, data traceability, audit, and operational transparency. Blockchain is an emerging technology that lacks in providing certain specifications and surety required to the business organizations to adopt the new technology. For instance, due to limited interoperability support, integrating blockchain-based systems with legacy systems is extremely challenging. Also, the lack of regulation, awareness, understanding, governance, and developers' support are some of the imperative challenges that should be addressed in the future.

Throughput and Latency: Blockchain nodes invest ample time and energy to arrange and validate the transactions, store them, and create and broadcast the block to other mining nodes for verification and updating. The throughput of the public blockchain platforms such as Bitcoin and Ethereum is a few transactions per second. Ethereum blockchain platform takes approximately 15 seconds to create a block; whereas, block time for Bitcoin platforms is 8 minutes. On the other hand, the private blockchain platforms such as Hyperledger Fabric and Besu completes several hundreds of transactions per second, thereby offering high throughput to the involved stakeholders. There is an urgent need to propose lightweight, attacks-resilient, and resource-efficient consensus algorithms to increase the throughput of existing blockchain platforms. Since our proposed system has considered the Ethereum blockchain platform, therefore the throughput of the system is dependent on the throughput of the underlying Ethereum blockchain platform.

Interoperability: The stakeholders involved in the forward supply chain and waste management of medical equipment and supplies could be practicing multiple platforms to do their business activities. The interoperability feature in existing blockchain-based solutions provides cross-platform communication ability to increase system productivity and throughput. Thus, the freedom from intermediaries (e.g., arbitration services) can enable efficient information sharing among the participating organizations [36], [37]. The difference in consensus protocols, implemented security schemes, registration services, governance model, and supported languages are the primary challenges in developing an interoperable platform. It is desired that the involved stakeholders in forward supply chain and waste management activities should be using the same blockchain platform, or the selected platform should have interoperability support for cross border communication. Our system is generic that can be customized for any platform with little effort since its code is publicly made available.

Scalability: In recent years, the transaction rate of many of the blockchain platforms including Ethereum has increased. This notable increase in blockchain transaction rate can influence the average response time of the blockchain, transaction fees, and the total amount of energy consumption. Consequently, the increased transaction rate can make the blockchain remarkably slow and unstable [38]. The complex, highly fragmented, and dynamic supply chain of medical equipment and supplies can generate an ample amount of data, thereby causing a delay in transactions execution time and throughput. In our proposed system, Algorithm 4 is the most expensive part as it has involved numerous state parameters and operations to periodically store the current location, waste state, estimated time to reach the destination, and other parameter's data on the ledger. To some extent, it can be further optimized by extending the time interval between two updates to generate and store less data on the blockchain. However, stretching the time interval can affect the reliability of the system. Highly scalable platforms that offer strong security features and throughput can be used for system deployment to enrich the user's experience.

On-chain and Off-chain Governance: Blockchain evolves and upgrades itself amidst ongoing developments to meet the innovative requirements of the highly competitive industries. Governance defines the mechanism of how decentralized technology can adapt to the changes and stay up to date to enrich the user's experience. Blockchain is governed and run by several communities including, core developers, node operators, token holders, and blockchain teams. Being an evolving technology, mature strategies that can efficiently govern blockchain are lacking. Broadly, governance models are classified into two types such as on-chain and off-chain blockchain governance. The Ethereum platform follows an offchain governance model. The on-chain governance model can leverage the voting mechanism to achieve direct democracy in blockchain affairs, such as the development of incentives and consensus protocols. Tezos and hard fork are on-chain and offchain governance strategies, respectively [39]. The consistently changing blockchain platforms, due to governance strategies, could affect the security and performance of the developed systems. Our proposed study has not considered this aspect, as the primary goal of this research is to develop and present a working prototype of the system, test it on the available public blockchain platform, and estimate its feasibility and adaptability to the healthcare organizations.

\section{Generalization}

This research proposes an Ethereum blockchain-based system that can efficiently trace and track the forward supply chain of medical equipment supplies related to COVID-19. It also assists in tracing and tracking the medical waste caused by medical equipment and supplies and assures that the medical shipped waste complies with COVID-19 safety rules. It employs sensors to monitor and ensure that sealed medical waste bags cannot be exposed during shipment to minimize the COVID-19 from spreading. It cancels the shipper license for violating the rules specifically designed to decrease the chances of COVID-19 from spreading during waste transportation. It also assures that medical waste found missing is immediately reported by the medical waste treatment facility.

COVID-19 inherits many similar characteristics of many other viruses or diseases that belong to a similar category (e.g., contagious and human-to-human transmittable). Since the proposed solution has successfully traced and tracked the forward supply chain and waste related to medical equipment and supplies; therefore, this research is equally valid to manage the forward supply chain of medical equipment for other diseases too. For example, the authorities can use this research 
to trace and track the medical supplies for Influenza (Flu). In addition to the forward supply chain and waste management of medical equipment and devices, this research is valid to trace and track any vaccine for COVID-19 treatment. The examples of other beneficiaries of this work include drug dealers, pharmacists, telehealth specialists, telemedicine, regularities, health authorities, and health professionals.

The smart contracts are implemented in solidity for the Ethereum-blockchain platform. Since our solution is generic; therefore, one can easily transform it with minimal efforts for other blockchain platforms. As our solution can successfully minimize the spread of COVID-19 during the forward supply chain and waste handling, hence healthcare authorities can extend it by introducing new laws to further minimize the spreading of COVID-19.

\section{CONCLUSION}

In this paper, we have designed, developed, and evaluated a blockchain-based solution to automate forward supply chain processes and establish data provenance about the COVID-19 medical equipment and their waste disposal. We developed four smart contracts and proposed five algorithms to implement all functionalities and trigger events and notifications. We integrated the Ethereum blockchain with decentralized storage of IPFS to provide a secure, transparent, auditable, reliable, traceable, and trustworthy solution for the forward supply chain and waste management of COVID-19 medical equipment. The proposed approach can assist authorities in assuring that the COVID-19 medical waste is disposed of properly, and COVID-19 testing centers are using genuine medical equipment to treat COVID-19 patients. We presented a detailed cost analysis to show the affordability of the proposed approach. We also evaluated the proposed approach against known vulnerabilities using the SmartCheck software. The proposed approach is generic and applicable to various use case scenarios with minimal modifications. Our implementation code is made publicly available on GitHub. In the future, we aim to design and implement an incentive system for the COVID-19 treatment hospitals to dispose of medical waste.

\section{ACKNOWLEDGMENT}

This publication is based upon work supported by the Khalifa University of Science and Technology under Awards No. CIRA-2019-001 and RCII-2019-002, Center for Digital Supply Chain and Operations Management.

\section{REFERENCES}

[1] M. L. Ranney, V. Griffeth, and A. K. Jha, "Critical supply shortages-the need for ventilators and personal protective equipment during the COVID-19 pandemic," New England Journal of Medicine, vol. 382, no. 18 , p. e41, 2020.

[2] Laura Wood, "Global Medical Waste Management Market (2020 to 2030) - COVID-19 Implications and Growth," [Online]. Accessed on 24/12/2020, August 2020, https://www.globenewswire.com/newsrelease/2020/08/12/2077096/0/en/Global-Medical-Waste-ManagementMarket-2020-to-2030-COVID-19-Implications-and-Growth.html.

[3] Peter J, "Blockchain powered environmental hazardous waste management systems for a more sustainable better earth and future," [Online]. Accessed on 15/11/2020, October 2019, https://pagarba.io/blockchain-powered-environmental-hazardous-wastemanagement-systems-for-a-more-sustainable-better-earth-and-future/.
[4] S. Saberi, M. Kouhizadeh, J. Sarkis, and L. Shen, "Blockchain technology and its relationships to sustainable supply chain management," International Journal of Production Research, vol. 57, no. 7, pp. $2117-$ 2135, 2019.

[5] R. W. Ahmad, K. Salah, R. Jayaraman, I. Yaqoob, S. Ellahham, and M. Omar, "Blockchain and COVID-19 pandemic: Applications and challenges," 2020, DOI: 10.36227/techrxiv.12936572.v1.

[6] I. Yaqoob, K. Salah, R. Jayaraman, and Y. Al-Hammadi, "Blockchain for healthcare data management: Opportunities, challenges, and future recommendations," Neural Computing and Applications, 2020, DOI: 10.1007/s00521-020-05519-w.

[7] S. Sahoo and R. Halder, "Blockchain-based forward and reverse supply chains for E-waste management," in International Conference on Future Data and Security Engineering. Springer, 2020, pp. 201-220.

[8] S. A. Abeyratne and R. P. Monfared, "Blockchain ready manufacturing supply chain using distributed ledger," International Journal of Research in Engineering and Technology, vol. 5, no. 9, pp. 1-10, 2016.

[9] M. D. LaGrega, P. L. Buckingham, and J. C. Evans, Hazardous waste management. Waveland Press, 2010.

[10] G. K. Shyam, S. S. Manvi, and P. Bharti, "Smart waste management using internet-of-things (IoT)," in 2nd international conference on computing and communications technologies (ICCCT). IEEE, Chennai, India, 2017, pp. 199-203.

[11] D. Misra, G. Das, T. Chakrabortty, and D. Das, "An IoT-based waste management system monitored by cloud," Journal of Material Cycles and Waste Management, vol. 20, no. 3, pp. 1574-1582, 2018.

[12] R. B. d. Silva and C. A. d. Mattos, "Critical success factors of a drug traceability system for creating value in a pharmaceutical supply chain (psc)," International journal of environmental research and public health, vol. 16, no. 11, p. 1972, 2019.

[13] C. K. M. Lee, M. N. Cheng, and C. K. Ng, "IoT-based asset management system for healthcare-related industries," International Journal of Engineering Business Management, vol. 7, no. Godište 2015, pp. 7-19, 2015.

[14] S. Aleyadeh and A.-E. M. Taha, "An IoT-based architecture for waste management," in International Conference on Communications Workshops (ICC Workshops). IEEE, Kansas City, MO, USA, 2018, pp. $1-4$.

[15] C. Tao and L. Xiang, "Municipal solid waste recycle management information platform based on internet of things technology," in International Conference on Multimedia Information Networking and Security. IEEE, Nanjing, Jiangsu, China, 2010, pp. 729-732.

[16] T. Bakhshi and M. Ahmed, "IoT-enabled smart city waste management using machine learning analytics," in 2nd International Conference on Energy Conservation and Efficiency (ICECE). IEEE, Lahore, Pakistan, 2018, pp. 66-71.

[17] J.-H. Tseng, Y.-C. Liao, B. Chong, and S.-w. Liao, "Governance on the drug supply chain via gcoin blockchain," International journal of environmental research and public health, vol. 15, no. 6, p. 1055, 2018.

[18] R. Kumar and R. Tripathi, "Traceability of counterfeit medicine supply chain through blockchain," in 11th International Conference on Coтmunication Systems \& Networks (COMSNETS). IEEE, Bengaluru, India, 2019 , pp. 568-570.

[19] X. Xia, X. Lin, W. Dong, and Z. He, "Design of traceability system for medical devices based on blockchain," in Journal of Physics: Conference Series, vol. 1314, no. 1. IOP Publishing, 2019, p. 012067.

[20] J. Pane, K. M. Verhamme, L. Shrum, I. Rebollo, and M. C. Sturkenboom, "Blockchain technology applications to postmarket surveillance of medical devices," Expert Review of Medical Devices, pp. 1-10, 2020

[21] M. Lamichhane, "A smart waste management system using IoT and blockchain technology," Tech. Rep, Master thesis, ITMO University, Russia., 2017.

[22] N. Gupta and P. Bedi, "E-waste management using blockchain based smart contracts," in International Conference on Advances in Computing, Communications and Informatics (ICACCI). IEEE, Bangalore, India, 2018, pp. 915-921.

[23] M. Poongodi, M. Hamdi, V. Vijayakumar, B. S. Rawal, and M. Maode, "An effective electronic waste management solution based on blockchain smart contract in 5G communities," in 3rd 5G World Forum (5GWF). IEEE, 2020, pp. 1-6.

[24] M. R. Laouar, Z. T. Hamad, and S. Eom, "Towards blockchainbased urban planning: Application for waste collection management," in Proceedings of the 9th International Conference on Information Systems and Technologies, Cairo Egypt, 2019, pp. 1-6.

[25] D. Schmelz, K. Pinter, S. Strobl, L. Zhu, P. Niemeier, and T. Grechenig, "Technical mechanics of a trans-border waste flow tracking solution based on blockchain technology," in 35th International Conference on 
Data Engineering Workshops (ICDEW). IEEE, Macao, 2019, pp. 3136.

[26] G. Ongena, K. Smit, J. Boksebeld, G. Adams, Y. Roelofs, and P. Ravesteyn, "Blockchain-based smart contracts in waste management: A silver bullet?" in Bled eConference, 2018, p. 19.

[27] I. Omar, M. Debe, R. Jayaraman, K. Salah, M. Omar, and J. Arshad, "Blockchain-based supply chain traceability for COVID-19 PPE," TechRxiv preprint, 2020.

[28] O. Akter, "Blockchain leveraged incentive providing waste management system," Preprint:RG.2020, 2020.

[29] T. K. Dasaklis, F. Casino, and C. Patsais, "A traceability and auditing framework for electronic equipment reverse logistics based on blockchain: The case of mobile phones," arXiv preprint arXiv:2005.11556, 2020.

[30] N. Nizamuddin, K. Salah, M. A. Azad, J. Arshad, and M. Rehman, "Decentralized document version control using ethereum blockchain and ipfs," Computers \& Electrical Engineering, vol. 76, pp. 183-197, 2019.

[31] H. R. Hasan and K. Salah, "Blockchain-based solution for proof of delivery of physical assets," in International Conference on Blockchain. Springer, Cham, 2018, pp. 139-152.

[32] H. R. Hasan, K. Salah, R. Jayaraman, J. Arshad, I. Yaqoob, M. Omar, and S. Ellahham, "Blockchain-based solution for COVID-19 digital medical passports and immunity certificates," IEEE Access, vol. 8, pp. 222 093-222 108, 2020.

[33] K. Baird, S. Jeong, Y. Kim, B. Burgstaller, and B. Scholz, "The economics of smart contracts," arXiv preprint arXiv:1910.11143, 2019.

[34] EtH Station, "ETH Gas Station," [Online]. Accessed on 22/12/2020, December 2020, https://ethgasstation.info/.

[35] Smart Check, "SmartCheck," [Online]. Accessed on 23/12/2020, December 2020, https://tool.smartdec.net/.

[36] Y. Lu, "Blockchain and the related issues: A review of current research topics," Journal of Management Analytics, vol. 5, no. 4, pp. 231-255, 2018.

[37] M. Herlihy, "Atomic cross-chain Swaps," in ACM proceedings of symposium on principles of distributed computing, 2018, pp. 245-254.

[38] R. W. Ahmad, H. Hasan, I. Yaqoob, K. Salah, R. Jayaraman, and M. Omar, "Blockchain for aerospace and defense: Opportunities and open research challenges," Computers \& Industrial Engineering, p. 106982, 2020.

[39] Rajarshi Mitra, "What is Blockchain Governance: Ultimate Beginner's Guide," [Online]. Accessed on 28/12/2020, December 2020, https://blockgeeks.com/guides/what-is-blockchain-governance-ultimatebeginners-guide/. 